\title{
Quelques aspects du niveau de richesse et de la vie matérielle des artisans de Québec et de Montréal, 1740-1755
}

\section{Jean-Pierre Hardy}

Volume 40, numéro 3, hiver 1987

URI : https://id.erudit.org/iderudit/304467ar

DOI : https://doi.org/10.7202/304467ar

Aller au sommaire du numéro

Éditeur(s)

Institut d'histoire de l'Amérique française

ISSN

0035-2357 (imprimé)

1492-1383 (numérique)

Découvrir la revue

Citer cet article

Hardy, J.-P. (1987). Quelques aspects du niveau de richesse et de la vie matérielle des artisans de Québec et de Montréal, 1740-1755. Revue d'histoire de l'Amérique française, 40(3), 339-372. https://doi.org/10.7202/304467ar

\section{Résumé de l'article}

L'étude vise à comparer le niveau de richesse et quelques aspects de la vie matérielle des artisans de Québec et de Montréal à l'aide principalement des inventaires après décès. Les fortunes mobilières révèlent qu'il existe dans une ville comme dans l'autre deux groupes d'artisans bien distincts : les plus démunis - près de $60 \%$ - avec moins de 700 livres en biens mobiliers, et les plus à l'aise dont la valeur des mêmes biens dépassent les 800 livres pour atteindre parfois 2000 livres. En ajoutant à ces biens le numéraire, les créances, les dettes et surtout les biens fonciers et immobiliers, il apparaît clairement que les fortunes les plus imposantes sont plus nombreuses dans la capitale. En effet, on y est moins nombreux à posséder sa maison, mais elle est généralement en pierre et vaut de trois à cinq fois plus que celle en bois de Montréal. On y possède aussi des pièces d'argenterie et du numéraire pour une valeur beaucoup plus considérable. Enfin, parmi les artisans qui peuvent investir, ceux de Québec préfèrent la maison de pierre alors que ceux de Montréal choisissent plutôt la terre, en général de moindre valeur.

La quantité, la qualité et la variété des biens mobiliers opposent beaucoup moins les artisans des deux villes que fortunés et moins fortunés. En effet, que l'on regarde le système de chauffage, la batterie de cuisine, la vaisselle, le mobilier de la salle commune ou de la chambre à coucher et les objets de décor, les écarts apparaissent frappants entre les deux groupes. Il n'y a évidemment rien de très surprenant à cette opposition entre riches et moins riches mais la comparaison a le mérite de dépeindre des intérieurs domestiques qui se distinguent à plusieurs égards en même temps que d'identifier les domaines de consommation privilégiés des plus à l'aise des artisans.
Ce document est protégé par la loi sur le droit d'auteur. L'utilisation des services d'Érudit (y compris la reproduction) est assujettie à sa politique d'utilisation que vous pouvez consulter en ligne.

https://apropos.erudit.org/fr/usagers/politique-dutilisation/ 


\title{
QUELQUES ASPECTS DU NIVEAU DE RICHESSE ET DE LA VIE MATÉRIELLE DES ARTISANS DE QUÉBEC ET DE MONTRÉAL, 1740-1755 ${ }^{1}$
}

\author{
JEAN-PIERRE HARDY \\ Division de l'histoire \\ Musée national de l'Homme
}

\begin{abstract}
RÉSUMÉ
L'étude vise à comparer le niveau de richesse et quelques aspects de la vie matérielle des artisans de Québec et de Montréal à l'aide principalement des inventaires après décès. Les fortunes mobilières révèlent qu'il existe dans une ville comme dans l'autre deux groupes d'artisans bien distincts: les plus démunis — près de $60 \%$ - avec moins de 700 livres en biens mobiliers, et les plus à l'aise dont la valeur des mêmes biens dépassent les 800 livres pour atteindre parfois 2000 livres. En ajoutant à ces biens le numéraire, les créances, les dettes et surtout les biens fonciers et immobiliers, il apparaît clairement que les fortunes les plus imposantes sont plus nombreuses dans la capitale. En effet, on y est moins nombreux à posséder sa maison, mais elle est généralement en pierre et vaut de trois à cinq fois plus que celle en bois de Montréal. On y possède aussi des pièces d'argenterie et du numéraire pour une valeur beaucoup plus considérable. Enfin, parmi les artisans qui peuvent investir, ceux de Québec préfèrent la maison de pierre alors que ceux de Montréal choisissent plutôt la terre, en général de moindre valeur.
\end{abstract}

La quantité, la qualité et la variété des biens mobiliers opposent beaucoup moins les artisans des deux villes que fortunés et moins fortunés. En effet, que l'on regarde le système de chauffage, la batterie de cuisine, la vaisselle, le mobilier de la salle commune ou de la chambre à coucher et les objets de décor, les écarts apparaissent frappants entre les deux groupes. Il n'y a évidemment rien de très surprenant à cette opposition entre riches et moins riches mais la comparaison a le mérite de dépeindre des intérieurs domestiques qui se distinguent à plusieurs égards en même temps que d'identifier les domaines de consommation privilégiés des plus à l'aise des artisans.

\begin{abstract}
By studying estate inventories, this article compares the levels of fortune and some aspects of material well-being of Québec and Montréal artisans. The study of moveable property reveals that in both cities artisans are divided into two groups: the poorest - almost 60\% of the total sample - who owned less than 700 livres of belongings, and the more prosperous whose estates contained between 800 and 2000 livres worth of this type of property. When money, debts and especially real estate are added it becomes clear that the largest fortunes are found in the colonial capital. Even though fewer Québec artisans owned a house, when they did it was normally a stone structure worth three to five times as much as a dwelling in Montréal. Money and silver were also more common in Québec. Finally, among those artisans who invested their assets, Québecers bought stone houses whereas Montréalers bought land which was generally worth less.
\end{abstract}

1 Nous remercions Nicole Castéran, John Dickinson et Thierry Ruddel pour leurs suggestions, Christine Chatré pour la collecte des données sur les artisans de Québec, ainsi que Danielle Forget pour la composition typographique. 
The quantity and quality of moveable property was similar in both cities and was dependent on total wealth. Whether it be in terms of heating equipment, kitchen utensils, dishes or furniture, striking differences opposed the more prosperous and the poorer members of this social group. This, in itself, is hardly surprising, but the study points out how the material environment differed and identifies consumer preferences among the wealthier artisans.

Les recherches entreprises depuis quelques années sur le niveau de richesse et la vie matérielle du 19e siècle québécois ont démontré la nécessité d'explorer davantage le siècle précédent. D'abord pour mieux cerner les antécédents de la vie matérielle du monde ouvrier; ensuite pour affiner les connaissances qui permettraient une meilleure distinction entre héritage français et apport britannique ${ }^{2}$. La littérature historique du $18 \mathrm{e}$ siècle qui touche au sujet est peu abondante et les descriptions de la vie matérielle sont souvent générales, parfois pittoresques mais rarement dirigées vers les groupes populaires urbains. Un sondage s'imposait donc dans les sources de la période française et particulièrement dans les inventaires après décès. Dans le cadre de cet article, nous nous restreignons au seul groupe des artisans de Montréal et de Québec et à la période 1740-1755. L'ampleur de la tâche exigée pour le seul dépouillement des inventaires nous dictait en effet ces contraintes. Ajoutons que l'insignifiance numérique des inventaires d'artisans pour Québec avant $1740^{3}$ et la poussée inflationniste qui gagne la colonie à la fin du Régime français et qui s'accentue sériesement vers les années 1755-1756 nous interdisaient d'étendre d'une ou deux décennies la période à l'étude ${ }^{4}$. Cet essai se veut donc un complément aux études déjà entreprises en même temps qu'un apport à une meilleure compréhension de la vie matérielle de l'époque.

L'inventaire après décès a été suffisamment exploité et critiqué en tant que document de base pour l'étude de la civilisation et de la mentalité pour qu'on ne s'y attarde pas icis. Mentionnons toutefois que sa représentativité, qui a été parfois mise en doute puis réhabilitée par des

2 Sur l'apport culturel britannique nous faisons allusion plus spécifiquement à notre article «Niveaux de richesse et intérieurs domestiques dans le quartier Saint-Roch à Québec, 1820-1850», Bulletin d' histoire de la culture matérielle (BHCM), 17 (printemps 1983): 63-94, et à celui de D. T. Ruddel, «The Domestic Textile Industry in the Region and City of Quebec, 1792-1832», BHCM, 17 (printemps 1983): 95-125.

3 Par exemple, entre 1730 et 1739 , nous n'avons repéré que trois inventaires de charpentiers, trois de menuisiers et un seul de maçons. Nous remercions Yvon Desloges de Parcs Canada de nous avoir fourni une première liste des inventaires des artisans à laquelle nous en avons ajouté quelques autres.

Voir par exemple les prix de certaines denrées entre 1755 et 1759 dans A. Shortt, Documents relatifs à la monnaie, aux changes et aux finances du Canada sous le Régime français, II: 904.

5 Pour une brève énumération des auteurs français, québécois et américains les plus connus qui ont utilisé et commenté l'inventaire après décès, voir l'article «Niveaux de richesse...», 92, notes 4 et 6 . 
sondages, ne fait pas encore l'unanimité ${ }^{6}$. Aussi convient-il d'aborder brièvement cet aspect de la méthodologie.

En 1744, la ville de Québec compte quelque 5000 habitants. Capitale administrative, siège des institutions religieuses et ville portuaire, ses principales activités économiques sont orientées vers le grand commerce d'import-export. A part la construction navale et, à un moindre degré, la tonnellerie, le secteur secondaire répond surtout à la demande locale. Les 422 gens de métier qu'on y dénombre représentent $8,4 \%$ de la population totale et entre 30 et $40 \%$ des chefs de ménage, pourcentages qui se maintiennent jusqu'au deuxième quart du $19 \mathrm{e}$ siècle. On y compte pas moins de 44 occupations différentes, affectées soit aux services soit à la transformation. Nous avons choisi d'étudier les métiers reliés à la transformation seulement et parmi ceux-ci, les sept métiers les plus populeux qui, d'après le recensement de 1744 , regroupent à eux seuls $70 \%$ de la main-d'oeuvre artisanale, apprentis non compris. Il s'agit, par ordre d'importance numérique, des charpentiers, des tailleurs d'habit, des menuisiers, des forgerons, des tonneliers, des cordonniers et des maçons. Un total de 39 inventaires entre 1740 et 1755 a pu être repéré et analysé. Les métiers sont inégalement représentés mais l'étude ne vise pas tant à établir une hiérarchie des métiers selon le niveau de richesse que de saisir l'ensemble du groupe ${ }^{7}$.

Les inventaires après décès de Montréal ont été tirés d'une liste exhaustive dressée par un groupe de recherche de l'Université de Montréal en vue d'effectuer un lexique des objets usuels trouvés dans les habitations de la Nouvelle-France ${ }^{8}$. Cette liste n'englobait que les indi-

6 Maurice Garden, dans «Les inventaires après décès: source globale de l'histoire lyonnaise ou juxtaposition de monographies familiales», Cahiers d' histoire, 12 (1967): 153-173, fut un des premiers à mettre en doute la représentativité de l'inventaire. Quant aux sondages qui s'opposent à Garden, mentionnons, pour la France, Adéline Daumard, «Structures sociales et classement socio-professionnel. L'apport des archives notariales au XVIIIe et au XIXe siècles», Revue historique $(R H), 86 \mathrm{e}$ année, 227, 1 (janvier-mars 1962): 139-154; Jean Meyer, La noblesse bretonne au XVIIIe siècle (Paris, École pratique des hautes études, Centre de recherche historique, 1966). Pour le Québec, Yvan Morin, «La représentativité de l'inventaire après décès. L'étude d'un cas: Québec au début du XIXe siècle», Revue d'histoire de l'Amérique française (RHAF), 34,4 (mars 1981): 515-533. L'auteur de cette enquête en arrive à démontrer que $11,3 \%$ des personnes majeures décédées à Québec entre 1800 et 1819 ont eu recours à un inventaire après décès, ce qui est légèrement supérieur à ce qu'on a noté en France, et que ces inventaires représentent bien la population quant à son âge moyen au décès, à sa religion, à son milieu géographique (quartier) et à son occupation. Fait à noter, même les milieux populaires y sont bien représentés.

Informations tirées de J.-P. Hardy et D. T. Ruddel, Les apprentis artisans à Québec, 1660-1815 (Montréal, Presses de l'Université du Québec, 1977), 4; F. Ouellet, Éléments d'histoire sociale au Bas-Canada (Montréal, Hurtubise HMH, 1972), 181 et 193. Louise Dechêne, "Quelques aspects de la ville de Québec au XVIIIe siècle d'après les dénombrements paroissiaux", Cahiers de géographie du Québec, 28,75 (décembre 1984): 484-490. Dans son analyse du recensement de 1744, l'auteure signale l'absence du statut professionnel dans $16 \%$ des cas de même qu'une imprécision dans les dénominations de métiers. Ces caractéristiques s'appliquant à toutes les couches de la société, elles n'affectent pas la valeur de notre base documentaire.

8 Il s'agit du livre de N. Genet, L. Décarie-Audet et L. Vermette, Les objets familiers de nos ancêtres (Montréal, Éditions de l'Homme, 1974), 304 p. Nous remercions Luce Vermette de nous avoir prêté cette liste de même que la plupart des inventaires photocopiés qui lui ont servi à écrire «Les feux domestiques à Montréal de 1740 à 1760». Thèse de maîtrise, Université Laval, $1977,180 \mathrm{p}$ 
vidus vivant à l'intérieur des murs entre 1740 et 1760 , excluant les faubourgs. Sur les 175 inventaires repérés représentant tous les groupes d'occupation, 46 concernaient les gens de métier. Après avoir éliminé tous ceux qui n'étaient pas reliés directement à la transformation ou ceux qui étaient associés trop étroitement au commerce (les bouchers et les aubergistes par exemple) de même que tous ceux de la période inflationniste 1756-1760, il restait 21 inventaires utilisables. Les occupations représentées sont sensiblement les mêmes qu'à Québec à l'exception des charpentiers et des tonneliers.

Montréal connaît le plus fort de son développement dans le premier tiers du 18e siècle puis semble stagner par la suite. De 1327 habitants qu'elle compte en 1707 , elle passe à 3575 en 1741 et à 4000 en 1754, soit une population presque deux fois moins nombreuse qu'à Québec à la même date. Le portrait le plus complet des activités urbaines et des groupes sociaux date des années 1731 et comme la croissance de la ville ralentit après cette date, on peut légitimement penser qu'il n'est pas très différent 20 ans plus tard. Les deux cinquièmes de la population active font partie des services administratifs, religieux, militaires et domestiques. Un tiers environ vit du commerce des fourrures et le secteur productif vient en dernier lieu avec seulement une cinquantaine d'artisans qui vivent du marché local ${ }^{9}$. Considérant que ces données valent pour la ville et les faubourgs, que notre base documentaire exclut les faubourgs et contient tous les inventaires disponibles, on est en droit de penser qu'elle est représentative de la gent artisanale.

L'âge du chef de famille est un autre aspect de la représentativité qui mérite attention. Dans les échantillons de Québec et de Montréal, une majorité d'artisans (67\%) se situe entre 40 et 60 ans, respectivement 16 et $17 \%$ entre 30 et 40 ans, et 16 et $15 \%$ au-dessus de 60 ans. Bien qu'il soit généralement convenu de ne considérer que les individus d'âge moyen ${ }^{10}$, nous n'avons pas jugé opportun d'éliminer les extrêmes qui façonnent et incarnent autant que les autres cette réalité que l'on veut saisir. Ajoutons que la structure des âges étant sensiblement la même à Québec et à Montréal, l'inclusion de toutes les couches n'interdit pas la comparaison entre les deux villes.

Ce minimum d'explication accordée à la source principale permet d'aborder avec moins d'incertitude le portrait général des artisans des deux villes. Établissons d'abord la hiérarchie des fortunes qui nous

\footnotetext{
9 Ce portrait est entièrement dû à Louise Dechêne, «La croissance de Montréal au XVIIIe siècle», RHAF, 27,2 (septembre 1973): 173-179.

${ }_{10}$ Voir, par exemple, les nombreux articles de J.-P. Wallot et Le peuple de Paris de D. Roche (Paris, Aubier-Montaigne, 1981). Ces deux auteurs jugent préférable d'éliminer les plus jeunes et les plus vieux, ceux-ci parce qu'ils n'ont pas eu le temps de s'établir et d'accumuler suffisamment de biens, ceux-là parce qu'au seuil de la vieillesse, ils ont peut-être dépensé leur avoir pour assurer leur retraite.
} 
procurera un premier instantané des similitudes et des différences. Dressons ensuite un bilan des possessions foncières et immobilières, à commencer par l'habitation, puis pénétrons dans les intérieurs domestiques. Nous devrions être en mesure d'évaluer certains aspects du confort ou de l'inconfort matériel qui était le leur au $18 \mathrm{e}$ siècle ${ }^{11}$.

\section{1 - LES ARTISANS: UN GROUPE HÉTÉROGENE}

L'historiographie situe généralement les artisans juste au-dessus des sans métier dans la hiérarchie sociale ${ }^{12}$ mais le groupe est loin d'être homogène, même à l'intérieur d'un métier. C'est que les artisans en Nouvelle-France ne connaissent ni jurandes ni système de maitrise tels qu'ils existent dans la métropole ${ }^{13}$. La porte est donc grande ouverte aux initiatives personnelles et à l'esprit d'entreprise que seules les conjonctures et les situations financières individuelles viennent renforcer ou apaiser. Ce trait caractéristique du monde artisanal a été bien démontré par Louise Dechêne pour le $17 \mathrm{e}$ siècle et le tournant du $18 \mathrm{e}$ siècle à Montréal. La population étant encore jeune et peu nombreuse, les exemples choisis, forcément réduits et trop épars, présentaient toutefois des écarts de fortune si considérables que le portrait d'ensemble était difficile à saisir ${ }^{14}$. Les inventaires retenus pour cette étude permettent donc à la fois de compléter ce portrait à une période ultérieure et de comparer Montréal à Québec. Pour ce faire, considérons d'abord la fortune mobilière, une valeur fort révélatrice du genre de vie puisque l'essentiel est composé d'objets qui meublent le paysage quotidien des artisans. Nous ajouterons ensuite le numéraire, les créances et les dettes, ces dernières reflétant souvent les avoirs fonciers et immobiliers. On obtiendra ainsi ce qu'il est convenu d'appeler la fortune mobilière nette de chaque famille ${ }^{15}$.

La moyenne globale des biens mobiliers est de 633 livres à Québec et de 927 livres à Montréal mais, comme toutes les moyennes, elles voilent une bonne partie de la réalité. A Québec, par exemple, aucune fortune ne se situe entre 500 et 800 livres (tableau 1). On y voit plutôt deux groupes qui s'opposent nettement: d'un côté les plus démunis qui

11 Sur la notion de confort relié à l'habitation, voir P. H. Chombart de Lauwe, La vie quotidienne des familles ouvrières (Paris, Éditions du CNRS, 1977), particulièrement le chapitre 3: Le logement, le ménage et l'espace familial; voir aussi Jean et Françoise Fourastié, Histoire du confort (Paris, Presses universitaires de France, coll. «Que sais-je?, no 449, 1973), 130 p.

${ }_{12}$ Voir, entre autres, L. Dechêne, Habitants et marchands de Montréal au XVIIe siècle (Paris, Plon, 1974), 393-394 et Marcel Trudel, Initiation à la Nouvelle-France (Toronto, Holt, Rinehart and Winston, 1968), 155-156.

13 Pour aller au-delà de cette simplification, voir J.-P. Hardy et D. T. Ruddel, Les apprentis..., 11-36.

Las auteure passe vite sur sa méthode de calcul des biens fonciers et immobiliers et il n'est pas touj.

15 Le terme est emprunté à J.-P. Wallot et G. Paquet mais il s'agit davantage de la situation financière des individus car la valeur des biens fonciers et immobiliers n'est pas ajoutée aux dettes. 
TABLEAU 1

Répartition des families en fonction

de la valeur des biens mobiliers ${ }^{1}$ et

de la fortune mobilière nette ${ }^{2}$

(en livres de 20 sols)

\begin{tabular}{|c|c|c|c|c|c|c|c|c|c|c|c|c|c|c|c|}
\hline & $\begin{array}{c}\text { Bilan } \\
\text { négatif }\end{array}$ & $\begin{array}{c}1 \\
\text { à } \\
99\end{array}$ & $\begin{array}{c}100 \\
\text { à } \\
199\end{array}$ & $\begin{array}{c}200 \\
\text { à } \\
299\end{array}$ & $\begin{array}{c}300 \\
\text { à } \\
399\end{array}$ & $\begin{array}{c}400 \\
\text { à } \\
499\end{array}$ & $\begin{array}{c}500 \\
\text { à } \\
599\end{array}$ & $\begin{array}{c}600 \\
\text { à } \\
699\end{array}$ & $\begin{array}{c}700 \\
\text { à } \\
799\end{array}$ & $\begin{array}{c}800 \\
\text { à } \\
899\end{array}$ & $\begin{array}{c}900 \\
\text { à } \\
999\end{array}$ & $\begin{array}{c}1000 \\
\text { à } \\
1299\end{array}$ & $\begin{array}{c}1300 \\
\text { à } \\
1599\end{array}$ & $\begin{array}{c}1600 \\
\text { à } \\
1999\end{array}$ & $\begin{array}{l}2000 \\
\text { et } \\
\text { plus }\end{array}$ \\
\hline $\begin{array}{c}\text { Québec } \\
1 \\
2 \\
\text { Montréal } \\
1 \\
2\end{array}$ & 13 & $\begin{array}{l}4 \\
1\end{array}$ & $\begin{array}{l}2 \\
3\end{array}$ & $\begin{array}{r}10 \\
4 \\
1\end{array}$ & $\begin{array}{l}4 \\
3\end{array}$ & 3 & $\begin{array}{l}3 \\
1\end{array}$ & 2 & $\begin{array}{l}1 \\
1\end{array}$ & $\begin{array}{l}5 \\
3\end{array}$ & 2 & $\begin{array}{l}5 \\
5 \\
4 \\
3\end{array}$ & $\begin{array}{l}2 \\
2 \\
3 \\
1\end{array}$ & $\begin{array}{l}2 \\
2 \\
2\end{array}$ & 5 \\
\hline
\end{tabular}


dominent (59\%) avec moins de 500 livres et de l'autre, ceux qui ont entre 800 et 2000 livres de biens mobiliers. Aucun artisan ne réussit à accumuler suffisamment de biens mobiliers pour dépasser en valeur la barre des 2000 livres. L'ajout du numéraire, des créances et des dettes aux biens mobiliers, loin de niveler ces fortunes, vient accentuer les écarts. La proportion des démunis demeure relativement stable à $61 \%$ mais parmi ceux-ci, plus de la moitié présente un bilan négatif, ce qui représente 33\% de l'ensemble des artisans. Chez les plus à l'aise le pourcentage baisse légèrement ( 41 à $38,5 \%$ ), mais cinq individus représentant $13 \%$ de l'ensemble se sont hissés en haut de 2000 livres, avec des fortunes respectives de $3239,3296,7510,8445$ et 11691 livres. Et c'est sans compter leurs possessions foncières et immobilières que nous aborderons plus loin.

La répartition des fortunes mobilières présente à Montréal une image légèrement différente: $52 \%$ ont entre 200 et 800 livres de biens mobiliers, $48 \%$ entre 900 et 2000 livres. On a l'impression d'être en présence d'un plus grand étalement des fortunes mais celle-ci est vite dissipée par l'addition du numéraire, des dettes et des créances. Riches et pauvres s'opposent en effet comme à Québec avec toutefois un peu plus de bilans négatifs $(38 \%)$ et un pourcentage à peu près semblable de fortunes supérieures à 2000 livres (14\%). Notons par contre que ces fortunes dépassent à peine les 2000 livres et qu'une seule, de 4549 livres, se compare avec celles de Québec ${ }^{16}$.

A quelques exceptions près, les plus fortunés à Québec se recrutent parmi les tonneliers, les maçons et les forgerons. A Montréal, c'est parmi les forgerons, les menuisiers et les cordonniers. Dans les deux villes, ces métiers semblent correspondre aux forces les plus vives de la production artisanale locale. La tonnellerie dans la capitale est une entreprise en bonne santé qui satisfait les divers besoins des exportations de marchandises et les maçons, nous le verrons plus loin, répondent aux exigences d'une ville qui se construit en pierre. A Montréal, les menuisiers jouent le rôle des maçons à Québec, les constructions en bois étant encore chose courante jusqu'au milieu du $18 \mathrm{e}$ siècle et les cordonniers tirent leur revenu du marché local certes mais aussi des tanneries, principales industries de l'endroit, avec lesquelles ils sont parfois associés. Parmi les plus démunis - majoritaires partout mentionnons les tailleurs des deux villes et les charpentiers de Québec.

Même opposition donc entre démunis et plus fortunés dans les deux villes, avec un contraste plus frappant à Québec, telle est la pre-

16 Il est certain que d'autres fortunes aussi considérables existent chez les artisans de Montréal mais elles semblent être davantage le produit d'un commerce que de l'activité professionnelle de l'artisan. Les artisans qui sont en même temps commerçants ou marchands ne sont pas considérés ici. Voir par exemple, le forgeron Paul Lemay dit Delorme qui a 3928 livres de biens mobiliers, 1602 livres de numéraires, aucune dette passive et 4800 livres de créances dues à son magasin général. Archives nationales du Québec à Montréal, Gr. J.-C. Panet, 24 septembre 1755. 
mière caractéristique du monde artisanal. Même opposition avons-nous dit, mais non pas même fortune et il est possible que les assises de cette richesse ne soient pas les mêmes. Les quelques fortunes importantes rencontrées à Québec, principalement le résultat du numéraire, des créances et des dettes, laissent en effet entrevoir des activités professionnelles et des tractations commerciales d'un ordre différent dans les deux villes. Aussi, est-il nécessaire, pour cerner de plus près cette réalité complexe, d'examiner et de confronter quelques-unes des principales composantes de ce patrimoine.

\section{2 - LES PRINCIPALES POSSESSIONS}

\section{A - L'habitation}

Jusqu'au premier quart du $18 \mathrm{e}$ siècle, Montréal est construite majoritairement en bois et ce n'est qu'après le grand incendie de 1721 que les constructions en pierre se multiplient pour atteindre $44 \%$ en 1731 et probablement un peu plus par la suite ${ }^{17}$. Un décompte effectué entre 1728 et 1760 donne en effet une proportion à peu près égale aux deux types de construction, soit $48,5 \%$ pour le bois et $50,2 \%$ pour la pierre $^{18}$. Toutefois, probablement parce que riches et pauvres vivent encore côte à côte de sorte que cabanes, maisons en bois et constructions en pierre se voisinent, l'image globale qui s'y dégage laisse au visiteur l'impression d'une ville où le bois domine. Pehr Kalm dira lors de son passage en 1748 que «quelques maisons de la ville sont construites en pierre mais la plupart le sont en bois de charpente mais très élégamment construites» ${ }^{19}$. L'habitation des artisans propriétaires qui servent à notre enquête répond davantage à l'image que Pehr Kalm nous a laissée de la ville qu'aux résultats des dernières recherches qui couvraient l'ensemble de la ville et des groupes sociaux. En effet, $74 \%$ d'entre eux possèdent une maison en bois contre $26 \%$ seulement en pierre (tableau 2).

A Québec, les proportions sont complètement inversées: $81 \%$ des propriétaires de nos inventaires ont choisi la pierre alors que le reste a opté pour le bois. Cela les situe tout près de la moyenne pour l'ensemble de la ville qui compte entre 1728 et $1760,73,8 \%$ de maisons en pierre et $24,6 \%$ en bois. La construction des maisons en pierre, dont la proportion n'était que de $27 \%$ avant $1728^{20}$ a été fortement encouragée par les législations interdisant les constructions en bois en vue de réduire

17 L. Dechêne, Habitants..., 263.

18 Entre 1660 et 1727 il y aurait $64,4 \%$ de maisons en bois contre $31,4 \%$ en pierre; entre 1728 et $1760,48,5 \%$ sont en bois et $50,2 \%$ en pierre. G.-P. Léonidoff, «L'habitat de bois en Nouvelle-France: son importance et ses techniques de construction», BHCM, 14 (printemps 1982): 20.

9 Pehr Kalm, Voyage de Kalm en Amérique (Montréal, T. Berthiaume, 1880), 55-56 (Mémoire de la Société historique de Montréal, livraison 7-8).

20 G.-P. Léonidoff, op. cit., 21-22. 
TABLEAU 2

Fortune globale comparée, 1740-1755

\begin{tabular}{|c|c|c|c|c|c|c|c|c|c|c|c|c|c|c|c|}
\hline & \multirow{2}{*}{$\begin{array}{c}\begin{array}{c}\text { Biens } \\
\text { mobiliers }\end{array} \\
\text { moyenne }\end{array}$} & \multicolumn{4}{|c|}{ Numéraire } & \multicolumn{2}{|c|}{$\begin{array}{c}\text { Richesse } \\
\text { mobilière } \\
\text { nette }\end{array}$} & \multicolumn{2}{|c|}{$\begin{array}{c}\text { Modes } \\
\text { d'occupation }\end{array}$} & \multicolumn{2}{|c|}{$\begin{array}{l}\text { Matériaux } \\
\text { des } \\
\text { résidences }\end{array}$} & \multicolumn{4}{|c|}{$\begin{array}{l}\text { Autres biens fonciers } \\
\text { et immobiliers }\end{array}$} \\
\hline & & $\begin{array}{c}\% \text { de } \\
\text { possédants }\end{array}$ & $\begin{array}{l}\text { Valeur } \\
\text { globale }\end{array}$ & Moyenne & + & - & Moyenne & Prop. & Loc. & Pierre & Bois & $\begin{array}{c}\text { No. de } \\
\text { possédants }\end{array}$ & $\begin{array}{c}\text { No. de } \\
\text { Terres }\end{array}$ & $\begin{array}{l}\text { No. d'Em- } \\
\text { placements }\end{array}$ & $\begin{array}{l}\text { No. de } \\
\text { Maisons }\end{array}$ \\
\hline Québec & $633 \#$ & $38,5 \%$ & $13,762 \#$ & $917 \#$ & $67 \%$ & $33 \%$ & 911\# & $64 \%$ & $36 \%$ & $81 \%$ & $19 \%$ & $41 \%$ & 6 & 6 & 11 \\
\hline Montréal & $927 \#$ & $14 \%$ & $2,947 \#$ & $982 \#$ & $62 \%$ & $38 \%$ & 739\# & $90 \%$ & $10 \%$ & $26 \%$ & $74 \%$ & $28,5 \%$ & 7 & 1 & 2 \\
\hline
\end{tabular}


les incendies et par la proximité autant que par l'abondance des carrières $^{21}$. Ajoutons que le rôle de capitale, de siège du gouvernement et de lieu de rassemblement des élites du pays a certainement favorisé cette transformation radicale du paysage architectural en si peu d'années.

Entre 1740 et 1755 , le prix des maisons dans les deux villes connaît des fluctuations importantes tant à cause de l'inflation que des spéculations. Il est donc impossible d'obtenir le prix moyen du marché pour chaque année. Toutefois les données recueillies dans les inventaires et quelques actes de vente indiquent que la maison en bois vaut entre 1500 et 2000 livres, alors que celle en pierre se situe aux environs de 4000 et 5000 livres. A Montréal par exemple, la maison de pièce sur pièce à un étage du maître ferblantier Antoine Beaumont dit Pistolet est évaluée à 2100 livres en 1743 et celle du menuisier Denis Jourdain dit Labrosse, en pierre à deux étages, vaut 4000 livres en $1744^{22}$. A Québec, la maison en pierre à deux étages mesurant 30 pieds par $32 \mathrm{du}$ charpentier Marseron est évaluée à 3300 livres en 1744 alors que celle du tonnelier Louis Paquet, aussi en pierre, vaut 6000 livres en $1750^{23}$. Les valeurs avancées ici ne constituent en aucune façon des moyennes, mais elles présument déjà de la différence de qualité et tout probablement du confort de ces deux types d'habitation sans parler des effets possibles sur l'accessibilité à la propriété.

C'est par la qualité, en effet, et conséquemment par le prix des maisons qu'on explique en bonne partie que $90 \%$ des artisans de Montréal soient propriétaires contre $64 \%$ seulement à Québec. A Montréal, c'est $20 \%$ au-dessus de la moyenne pour l'ensemble de la ville, faubourgs compris ${ }^{24}$, alors qu'à Québec c'est quelque $30 \%$ au-dessus de la moyenne si on se fie aux plus récentes recherches sur la propriété à Québec qui évaluent à environ $33 \%$ les chefs de ménage propriétaires ${ }^{25}$. Ces pourcentages tirés de nos inventaires ne constituent évidemment que des ordres de grandeur mais ils n'en sont pas moins révélateurs de la place qu'occupent les artisans des deux villes dans la hiérarchie sociale.

Ces propriétaires se recrutent dans toutes les occupations à Montréal comme à Québec. N'insistons pas sur la pauvreté des quelques locataires de Montréal (10\%); la valeur de leurs biens mobiliers est deux fois moindre que la moyenne générale et ils ne possèdent aucun autre

\footnotetext{
${ }^{21}$ Au sujet des carrières dans la ville et aux environs, voir A. Charbonneau, Y. Desloges, M. Lafrance, Québec ville fortifiée du XVIIe au XIXe siècle (Québec, Éditions du Pélican, Parcs Canada, 1982), 224-226 et 248 ss.

22 ANQ-M, Gr. F. Simonet, 16 janvier 1743 et Gr. J.-C. Dufresne, 22 décembre 1744.

23 ANQ, Gr. J.-C. Panet, 29 juillet 1744 et A.-J. Saillant, 30 mai 1750.

24 Selon L. Dechêne, «Croissance...», 169, 70\% des ménages de l'agglomération seraient propriétaires en 1741 .

${ }_{25}$ Yvon Desloges de Parcs Canada (Québec) effectue présentement cette étude qui s'intitulera «Vivre à Québec, 16910-1749».
} 
avoir. Le portrait est légèrement différent dans la capitale: la majorité des locataires sont charpentiers et leur inventaire présente soit un bilan négatif, soit une fortune globale moyenne de 300 livres. Fortune et propriété sont donc intimement liées chez les artisans comme ailleurs.

Une plus grande accessibilité à la propriété de même que des biens mobiliers dont la valeur est supérieure à celle de Québec semblent conférer d'emblée à Montréal un certain avantage. Gardons-nous toutefois des apparences car la valeur des habitations à Québec non seulement nivelle certaines fortunes mobilières nettes mais renverse la situation d'ensemble au profit de cette ville. En effet, en ajoutant à la moyenne des fortunes mobilières nettes des deux villes les valeurs approximatives de 1500 livres pour les maisons en bois et de 4000 livres pour les maisons en pierre, une évaluation conservatrice, la fortune moyenne s'élève à 2765 livres à Montréal et à 4435 livres à Québec. Mais il n'y a pas que la valeur des habitations qui diffère d'une ville à l'autre, on y vit aussi dans des univers différents qui affectent la qualité de la vie. Voyons de plus près ce qui en est.

\section{La maison de bois}

La maison en bois d'environ 18 par 20 pieds, à un seul étage et contenant le plus souvent une seule pièce, domine le paysage domiciliaire de Montréal jusqu'au premier quart du 18e siècle. Vers 1740 , à tout le moins parmi les artisans, elle fait graduellement place à une maison plus spacieuse. L'ancienne maison dite de colonisation existe encore, mais l'artisan l'habite de moins en moins et préfère la vendre, l'agrandir ou encore la louer à plus démuni. Tel est le cas, entre autres, du maçon Jean-Baptiste Payet dit Saint-Amour qui délaisse sa vieille maison de pièce sur pièce de 17 pieds de long sur 20 pieds de large pour habiter tout à côté, rue Saint-Jacques, une maison de pierre plus spacieuse $^{26}$. Avec une telle maison, Payet compte parmi les exceptions à Montréal et son métier n'est peut-être pas étranger à la situation. En effet, nous l'avons déjà dit, c'est plutôt une maison en bois que l'artisan montréalais habite et elle n'a pas les dimensions aussi modestes que le laisse entendre la littérature; elle mesure parfois 40 par 30 pieds, surtout lorsqu'une boutique y est annexée, mais le plus souvent 30 par 25 pieds $^{27}$. Elle est basse, n'a qu'un seul étage ${ }^{28}$ et comprend deux ou trois pièces aux dimensions fort inégales. De moins en moins souvent trouvet-on de ces maisons à pièce unique de sorte que la subdivision de l'habitation n'est plus le seul privilège des couches aisées qui vivent en ce

26 ANQ-M, Gr. L.-C. Danré de Blanzy, ler décembre 1741. Pour un autre exemple de petite maison de 15 par 15 pieds, voir Gr. F. Simonet, 31 août 1745.

27 Pour les dimensions des maisons en bois, voir par exemple les greffes de F. Simonet, 2 décembre 1740 et 16 janvier 1743 ; J.-B. Adhemar, 23 novembre 1741 et 9 août 1747 . Les dimensions données sont les suivantes: 48 par 22, 36 par 25, 30 par 26, 30 par 22 pieds.

28 Seulement $4,48 \%$ des maisons en bois de Montréal possèdent un étage au-dessus du rezde-chaussée. G.-P. Léonidoff, op. cit., 23. 
domaine à l'heure de l'Europe ${ }^{29}$. De nombreux exemples attestent de ces changements dans les intérieurs domestiques. La maison du menuisier Jean-Baptiste Petit, qui mesure 30 par 26 pieds, comprend quatre pièces: deux chambres et deux cabinets ${ }^{30}$. Certes les cabinets sont petits et ne servent parfois qu'au rangement et une des deux chambres sert à la fois de cuisine et d'atelier de travail mais il reste suffisamment de place pour isoler les deux enfants mineurs et se retirer dans une autre chambre quand vient le temps du coucher. Tout le monde ne participe évidemment pas à un tel confort car les petites maisons sont encore nombreuses, qui forcent à l'encombrement et à la promiscuité. Voyez celle du cordonnier René Douillard de Montréal qui partage avec sa femme et ses quatre enfants mineurs un espace de 15 pieds par 15 divisé en une chambre et une cuisine servant aussi d'atelier. Ces quelques exemples, puisés parmi les plus modestes des artisans propriétaires, témoignent aussi bien des changements en cours que des aspirations des plus humbles vers le mieux-être des plus nantis.

\section{La maison de pierre}

Les données tirées des inventaires et des marchés de construction révèlent que les dimensions des maisons urbaines en pierre diffèrent peu d'une ville à l'autre. L'habitation dont la superficie dépasse les 1000 pieds carrés est généralement hors de portée des artisans et appartient plutôt aux marchands, aux militaires et aux quelques professionnels ${ }^{31}$. Les artisans les plus prospères vivent dans une maison à deux étages mesurant entre 700 et 800 pieds carrés et la majorité se contente de plus petit ${ }^{32}$. Notons toutefois que dans la capitale, les maisons en pierre de plus d'un étage sont légèrement plus nombreuses qu'à Montréal de sorte que la surface d'habitation s'en trouve accrue. A Montréal, par exemple, $51,5 \%$ des maisons en pierre ont un étage audessus du rez-de-chaussée alors qu'à Québec, $50,6 \%$ en ont un et 7,9\% en ont deux. Retenons la description de la maison du menuisier Bazile Marois de Québec que nous livre son inventaire; elle apparaît à plusieurs égards représentative de la maison de l'artisan propriétaire:

29 On sait que le $18 \mathrm{e}$ siècle européen voit se généraliser parmi les élites une nouvelle conception de l'espace qui tend à valoriser la vie familiale et l'intimité par opposition à la vie sociale. La maison des plus aisés se subdivise graduellement en plusieurs pièces dont chacune sert à des fonctions spécifiques. Sur les changements dans la conception de l'espace en relation avec la notion de famille, voir, entre autres, Philippe Ariès, L'enfant et la vie familiale sous l'ancien régime (Paris, Seuil, 1973). Peter Laslett, ed., Household and Family in Past Time (Cambridge University Press, 1972), Lawrence Stone, The Family, Sex and Marriage in England, 1500-1800 (New York, Harper and Row, 1977). Edward Shorter, Naissance de la famille moderne (Paris, Seuil, 1977).

ANQ-M, Gr. F. Simonet, 2 décembre 1740

31 L. Vermette, op. cit., 24-25 et N. Genet, «Habitation et aménagement intérieur à Montréal au milieu du XVIIIe siècle». Thèse de maîtrise (Arts et traditions populaires), Université Laval, 1977.

32 C'est le cas des quelques artisans de Montréal qui possèdent une maison en pierre et de tous ceux de Québec. Les climensions les plus fréquentes sont les suivantes: 26 par 30, 24 par 30, 20 par 34, 30 par 28. 
Sur un emplacement, contenant 24 pi. de front sur 26 pi. de profondeur, est construite une maison en pierre à 2 étages de 24 pieds de long sur 20 de large, avec cave et grenier... consistant au premier étage du rez-de-chaussée en une chambre et une allée en laquelle chambre sont 2 croisées sur le devant garnies de leur vitre... et une croisée derrière..., trois portes et une armoire dans la muraille, le tout garni de leurs ferrures, clef et loquets et un dressoir aussi dans la muraille garni de ses tablettes et un fond. Le plancher haut et bas double et au second étage une chambre à 3 croisées sur le devant et une sur le derrière... une petite cuisine dans laquelle est une cheminée et un dressoir dans la muraille... un petit garde manger et une petite armoire tenant à fer et à clous dans la cloison... les cloisons en bois de pin... deux portes garnies de leurs serrures et clefs... une galerie sur le derrière en bois de charpente... couverte en planches, un grenier fermant à clef avec deux lucarnes... une cour derrière de 24 pi. de long sur 6 de profondeur dans laquelle cour sont des latrines. ${ }^{33}$

Cette description permet de dégager certains avantages de la maison en pierre sur celle en bois. D'abord la solidité: celle-ci repose le plus souvent sur des soles en terre de sorte que la structure est laissée aux caprices des gels et dégels du printemps, et la cave, plutôt rare, n'a guère plus de quatre à cinq pieds de hauteur; celle-là est bien assise sur une fondation en pierre assez profonde pour servir de cave que d'aucuns utilisent comme atelier de travail. Ensuite l'espace: les deux types d'habitat auraient sensiblement le même nombre de pièces, trois en moyenne ${ }^{34}$, mais celui de Québec est presque deux fois plus spacieux grâce à l'ajout d'un deuxième étage. Et cet espace peut être utilisé à son maximum par l'utilisation d'un mode de rangement mural armoires ou dressoirs encastrés dans l'épaisseur du mur - que les structures de pièce sur pièce ne permettent que rarement. Enfin, l'enduit crème des murs intérieurs vient accentuer la clarté qui pénètre par les nombreuses croisées.

Avec un endroit pour cuisiner, parfois un pour travailler ${ }^{35}$ et un autre pour la vie familiale et sociale, on est loin de l'entassement que connaissent les artisans de certaines grandes villes françaises à la même époque où la «spécialisation des pièces est un luxe» ${ }^{36}$. A raison de 4,5

33 ANQ, Gr. C.-H. Dulaurent, ler août 1744.

34 Cette moyenne tirée des inventaires après décès des deux villes comprend autant les maisons en bois qu'en pierre. Elle mérite d'être confrontée avec d'autres documents tels les marchés de construction. En incluant les métiers de service tels que bouchers et boulangers dont les maisons ont souvent 5 et 6 pièces, L. Vermette arrive à une moyenne de 3,7 pièces à Montréal. L. Vermette, «Les feux...», 24.

35 A l'exception du forgeron dont la boutique est, soit dans la cour soit dans la cave, celle des autres artisans fait encore partie intégrante de la maison bien qu'elle occupe de plus en plus souvent une pièce à elle seule au rez-de-chaussée. Pour de nombreux exemples de boutiques isolées des activités domestiques, voir N. Genet, op. cit., 140 ss.

Voir par exemple, D. Roche, Le peuple de Paris, 120 et les auteurs cités à la note 29. 
personnes par habitation à Montréal et 4,4 à Québec ${ }^{37}$, les artisans propriétaires qui ne louent aucune partie de leur maison jouissent d'un espace familial que leur niveau de fortune ne pouvait laisser présager.

\section{B - Les autres biens immobiliers et fonciers}

Le patrimoine immobilier ne se limite pas uniquement à l'habitation: c'est aussi la deuxième maison, le terrain en ville ou la terre à la campagne. L'examen rapide des créances et des dettes, résultat en grande partie des investissements fonciers et immobiliers, révèle déjà des différences notables entre les deux villes. La nature de ces investissements nous en dit davantage.

Autant la formation géologique de Québec et son rôle de capitale ont pu influencer l'architecture domiciliaire, autant le caractère rural de Montréal a pu conditionner les investissements des artisans. Parmi les possibilités qu'offre le marché, ce sont en effet des terres aux alentours de la ville que les artisans montréalais choisissent d'acheter. La maison en bois semble présenter peu d'intérêt à l'investisseur soucieux d'un bon rendement, et les constructions nouvelles, peu nombreuses et surtout de pierre ${ }^{38}$ n'offrent guère plus d'attrait ou leur sont simplement inaccessibles à cause de leur valeur. Reste donc la terre de sorte que tous les individus $(28,5 \%)$ qui peuvent investir en possèdent au moins une aux environs de la ville. La superficie de ces terres varie entre 50 et 90 arpents avec une exception de 160 arpents et on ne trouve aucune accumulation importante chez ces artisans. Les forgerons, un groupe qui gagne bien sa vie à Montréal, se distinguent toutefois des autres en accaparant cinq des sept terres inventoriées ${ }^{39}$. C'est évidemment bien peu pour une ville comme Montréal à cette époque et il semble que dans l'ensemble, peu d'artisans s'intéressent au marché foncier et immobilier. La ville étant d'abord la plaque tournante du commerce des fourrures, les plus fortunés des artisans comme, par exemple P. Lemay, lorgnent plutôt du côté du commerce ${ }^{40}$.

Il est difficile d'évaluer tous ces biens mais les quelques informations que nous livrent les inventaires donnent de 900 à 1100 livres

37 Notre calcul a été effectué à partir des inventaires et exclut les enfants majeurs. Il ne s'agit donc pas des ménages moyens. En 1744, dans le secteur secondaire à Québec, le ménage moyen se compose de 5 personnes incluant apprentis et domestiques qui comptent pour $0,3 \%$ seulement. Y. Desloges, «Vivre à Québec, 1660-1749».

38 L. Dechêne, «La croissance...», 169.

39 Parmi ceux-ci, signalons Jean-Baptiste Trutteau, avec sa terre de 160 arpents dont 6 sont de «terre à la charrue» et Joseph Sénécal, avec sa terre à la côte Saint-Léonard qui comprend un cheptel évalué à 222 livres. Ils font partie de ces propriétaires ruraux habitant la ville et essayant tant bien que mal d'exploiter leurs biens en vue d'une rentabilité éventuelle. Voir à ce sujet L. Dechêne, Habitants..., 286 ss.

40 A l'instar du forgeron Paul Lemay, le tailleur Joseph Durocher, mieux connu comme marchand, est de ceux-là pour qui le magasin rempli de marchandises de traite rapporte beaucoup plus que le métier. Pour Durocher, voir ANQ-M, Gr. F. Simonet, 30 mai 1743 et Dictionnaire biographique du Canada, III. Pour Lemay dit Delorme, voir Gr. J.-C. Panet, 24 septembre 1755. 
pour les terres de 50 à 60 arpents et autant, parfois plus, pour les emplacements avec petite maison de bois en ville ${ }^{41}$. Ces données, bien qu'approximatives, facilitent la comparaison avec la capitale.

A Québec, les investissements se dintinguent à plusieurs égards. D'abord, un plus grand nombre y participe, 16 sur 39 (41\%); ensuite, c'est l'immobilier qui attire les artisans et non les terres comme à Montréal. Moins accessibles de la ville, les terres sont en effet peu convoitées si l'on considère que leurs propriétaires, dans trois cas sur cinq, comptent parmi les plus pauvres ${ }^{42}$. Ceux-ci sont d'ailleurs les seuls locataires ayant quelque bien foncier, ce qui laisse supposer qu'il s'agit davantage d'héritage familial que d'investissement réfléchi. Les emplacements en ville ou en banlieue ne sont pas plus nombreux que les terres malgré leurs prix qui n'apparaissent pas exorbitants, entre 500 et 800 livres, dépendant de leur localisation ${ }^{43}$. Seuls les plus fortunés toutefois peuvent s'en permettre l'acquisition et ils donnent lieu à un seul cas de spéculation, digne de mention pour un artisan. Il s'agit du terrain du cordonnier Joachim Girard de 19 arpents de superficie qui lui rapporte 440 livres de rente annuelle provenant des 19 habitants qui y sont établis. Girard a pour 1280 livres de biens mobiliers et sa succession inscrit plus de 5000 livres au passif mais il ne fait pas pour autant partie du groupe des démunis, bien au contraire. En plus d'une spacieuse résidence en pierre de deux étages comprenant six pièces et une boutique, il en possède une seconde, en moins bon état il est vrai, mais qui lui procure un revenu de location ${ }^{44}$. Les autres maisons en pierre à deux étages, ne valant pas moins de 4000 livres et atteignant parfois 7000 livres, sont la propriété des plus à l'aise des tonneliers, des forgerons et des maçons.

D'autres artisans de Québec investissent probablement davantage dans l'immobilier; nos inventaires qui s'étalent sur une quinzaine d'années seulement ne peuvent les atteindre tous. Les plus connus des entrepreneurs maçons par exemple, tels les Janson dit Lapalme, les Deguise dit Flamant, les Routhier et bien d'autres nous échappent ${ }^{45}$. Les quelques cas signalés illustrent toutefois que Québec, à l'inverse de Montréal, offre une conjoncture urbaine plus favorable sinon à la spéculation du

41 Voir, par exemple, les inventaires suivants: ANQ-M, Gr. Danré de Blanzy, 14 mai 1742; G. Dufresne, 4 mai 1740 et 2 décembre 1744 . Sur le prix des terres de l'île de Montréal au tournant du $18 \mathrm{e}$ siècle, en général de peu de valeur et n'attirant guère la spéculation, voir L. Dechêne, Habitants..., 242 ss et 286 ss.

42 Les trois n'ont qu'entre 204 et 265 livres de biens mobiliers.

43 Voir par exemple, ANQ, Gr. C. Barolet, 24 avril 1752 et Gr. B. De Godefus, 23 mars 1744.

44 ANQ, Gr. J. Pinguet, 10 juin 1745. Voir aussi le cas du menuisier François-Louis Borgia Levasseur. Son patrimoine comprend sa propre résidence en pierre, une autre à deux étages de 25 par 30 pieds, le cinquième d'une autre semblable provenant d'un vieil héritage, deux maisons en bois qui lui rapportent chacune 200 livres par année et une terre à la sortie de la ville, côte SaintJean, de 39 arpents. ANQ, Gr. J. Pinguet, 3 septembre 1744.

45 A. Charbonneau, Y. Desloges, M. Lafrance, Les fortifications..., 243 ss. 
moins à l'investissement. Ceux qui choisissent d'investir dans une deuxième maison de pierre en font foi. Ces mêmes exemples caractérisent aussi assez bien le patrimoine foncier et immobilier des artisans des deux villes en même temps qu'ils annoncent la supériorité de la fortune globale des artisans québécois. La valeur moyenne des biens meubles n'annonçait évidemment pas un tel constat même si on pouvait déjà y déceler une première indication dans la fortune mobilière nette (911 livres à Québec contre 739 livres à Montréal).

Un bref examen des créances et des dettes vient confirmer sinon la supériorité des fortunes, à coup sûr celle du volume des transactions. Voyons d'abord l'actif. A Montréal, la moyenne est de 270 livres par individu contre 669 livres à Québec. Dans la première ville, une seule créance dépasse les 700 livres, celle du menuisier Jourdain-Labrosse, et la majeure partie provient de sommes que ses fils Charles et Paul doivent à la succession ${ }^{46}$. Les autres créances se composent essentiellement de travaux ou de fournitures non payés ${ }^{47}$. A Québec, huit créances (20,5\% de l'ensemble) dépassent les 700 livres et parmi celles-ci les plus importantes s'élèvent à quelque 5000 - 6000 livres. Le document ne nous livre pas l'origine de toutes ces créances mais dans l'ensemble, il ressort clairement que les transactions de toutes sortes, souvent reliées au métier, y sont plus imposantes qu'à Montréal. A seul titre d'exemple, sur les 6328 livres du forgeron Morin dit Chenevert, 60\% nous est inconnu mais le reste provient de la vente d'une maison et «d'ouvrages faits pour le compte du Roy» ${ }^{48}$.

Le passif parle davantage des transactions foncières et immobilières. A Montréal, la moyenne est de 599 livres contre 744 livres à Québec. La différence n'est évidemment pas énorme mais les sommes ne sont pas engagées pour les mêmes raisons. A Montréal, sur les huit débiteurs $(38 \%)$ de plus de 800 livres, deux seulement ont une autre propriété que leur résidence; il s'agit du forgeron Étienne Campeau et du maçon Jean-Baptiste Payet ${ }^{49}$. C'est dire ici que l'essentiel des dettes va soit à la première propriété, soit aux menus travaux. A Québec, sur les 13 dettes de plus de 800 livres, au moins huit proviennent de rentes à payer sur une deuxième propriété. Dans l'ensemble donc, créances et dettes sont davantage reliées à une seconde propriété à Québec et témoignent également d'activités plus lucratives.

\section{C - Le numéraire}

Trente-huit et demi pour cent des artisans québécois contre quatorze pour cent seulement à Montréal réussissent à accumuler dans leurs

ANQ-M, Gr. Dufresne, 2 décembre 1744.

A titre d'exemple, 31 débiteurs se partagent la créance de 582 livres du cordonnier Alexis

Dubois. ANQ-M, Gr. J.-B. Adhemar, 9 décembre 1748.

48 ANQ, Gr. J.-C. Panet, 30 mars 1752.

49 ANQ-M, Gr. Dufresne, 4 mai 1740 et Gr. L.-C. Danré de Blanzy, ler décembre 1741. 
réserves des pièces sonnantes, des cartes et des ordonnances. La moyenne à Québec est de 917 livres, ce qui constitue une somme supérieure à la valeur moyenne des biens mobiliers de l'ensemble des artisans (633 livres). Bon nombre d'entre eux (6 sur 15) possèdent des sommes minimes en apparence, 60 à 200 livres mais elles haussent quand même de 20 à $40 \%$ leur avoir mobilier. Ceux-ci ont évidemment rarement de l'argenterie et encore moins des biens fonciers ou immobiliers autres que leur résidence. D'autres, par contre, les plus riches, ceux-là mêmes qui possèdent l'argenterie accumulent aussi du numéraire. Les forgerons Lemire et Morin dit Chènevert en ont respectivement pour 1000 et 2061 livres, ce qui dans le cas du dernier représente plus de deux fois son avoir mobilier (900 livres). Quant au maçon Jean Boucher dit Belleville, il se distingue nettement de la masse avec 5888 livres de billets, de louis d'or et d'écus, ce qui représente sept fois la valeur de ses biens mobiliers ${ }^{50}$.

On thésaurise aussi à Montréal, mais il ne semble pas que les revenus professionnels le permettent autant qu'à Québec ${ }^{51}$. Trois individus seulement affichent du numéraire et un seul, le cordonnier Alexis Dubois, en possède pour une valeur supérieure (2 600 livres) à son avoir mobilier (1 367 livres) ${ }^{52}$. Les deux autres n'en ont que pour 37 et 310 livres. Si le numéraire consigné dans les inventaires correspond à la réalité - il est aisé de soustraire de l'argent à la vue des estimateurs il y a tout lieu de supposer que la tendance à l'économie et à la thésaurisation chez les plus riches des artisans soit moins forte à Montréal qu'à Québec. Reflet de deux attitudes différentes face à l'argent, au capital? Sans doute que non; d'ailleurs les artisans ne sont pas ceux qui possèdent le capital dans cette société où le commerce est le moteur de l'économie. Simple reflet d'une ville qui favorise plus que l'autre l'enrichissement des artisans et l'accumulation des biens de toutes sortes? Probablement. Les investissements fonciers et immobiliers examinés plus haut ont en tout cas plaidé en faveur de cette idée.

Dans l'ensemble donc, les fortunes sont plus élevées et plus nombreuses dans la capitale et les habitations, plus coûteuses, apparaissent plus confortables. Reste à savoir de quelle façon cette aisance apparente se reflète dans la vie de tous les jours. Nous avons déjà une idée de la structure des intérieurs; il nous faut maintenant y pénétrer.

\section{3 - LES INTÉRIEURS DOMESTIQUES}

On a vu précédemment que la seule richesse mobilière des artisans montréalais dépassait de $46 \%$ celle de Québec. Les quelques salaires

50 ANQ, Gr. J.-C. Panet, 30 mars 1752 et 30 juillet 1742; Gr. C. Barolet, 3 mai 1745. (Dans l'ordre d'énumération dans le texte).

51 Le forgeron Lemay dit Delorme possède pour 1200 livres en numéraire et sa fortune mobilière s'élève à 3928 livres mais il est davantage commerçant qu'artisan. ANQ-M, Gr. P. Panet, 24 septembre 1755 .

52 ANQ-M, Gr. J.-B. Adhemar, 9 décembre 1748. 
TABLEAU 3

Structure de la richesse mobilière, $1740-1755$

\begin{tabular}{|c|c|c|c|c|c|c|c|c|c|c|c|c|c|c|c|c|c|c|c|c|c|c|c|}
\hline \multirow[b]{2}{*}{ Québec } & \multicolumn{2}{|c|}{${ }^{1} \quad 1 \%$} & \multicolumn{2}{|c|}{$\#^{2} \%$} & \multicolumn{2}{|c|}{$\#^{3} \%$} & \multicolumn{2}{|c|}{$\#^{4} \%$} & \multicolumn{2}{|c|}{${ }^{5} \%$} & \multicolumn{2}{|c|}{${ }^{6} \%$} & \multicolumn{2}{|c|}{${ }^{7} \%$} & \multicolumn{2}{|c|}{${ }^{8} \%$} & \multicolumn{2}{|c|}{${ }^{9} \%$} & \multicolumn{2}{|c|}{$\#^{10} \%$} & \multicolumn{2}{|c|}{${ }^{11} \%$} & \multirow{2}{*}{$\begin{array}{c}\begin{array}{c}\text { Moyenne } \\
\#\end{array} \\
633\end{array}$} \\
\hline & 212 & 33,5 & 155 & 24,5 & 22 & 3,4 & 60 & 3,5 & 7 & 1,1 & 56 & 8,8 & 59 & 9,3 & 3 &, 5 & 15 & 2,4 & 32 & 5,1 & 11 & 1,8 & \\
\hline Montréal & 252 & 27,1 & 232 & 25 & 31 & 3,3 & 86 & 9,3 & 1 &, 1 & 87 & 9,4 & 86 & 9,3 & 14 & 1,5 & 45 & 4,8 & 64 & 6,9 & 29 & 3,1 & 927 \\
\hline
\end{tabular}

1. Équipement domestique; 2. Mobilier; 3. Linge de maison; 4. Vêtements; 5 . Objets personnels; 6. Outils et instruments de travail; 7. Stock relié au métier; 8. Transport, moyens et accessoires; 9 . Animaux; 10. Denrées; 11. Divers.

TABLEAU 4

Équipement domestique: comparaison des valeurs et des quantités moyennes par famille, 1740-1755

\begin{tabular}{|c|c|c|c|c|c|c|c|c|c|c|c|c|c|c|}
\hline & \multicolumn{2}{|c|}{$\begin{array}{l}\text { I } \\
\text { Équipement } \\
\text { domestique }\end{array}$} & \multicolumn{2}{|c|}{$\begin{array}{c}\text { A } \\
\text { Éclairage }\end{array}$} & \multicolumn{2}{|c|}{$\begin{array}{c}\text { B } \\
\text { Chauffage }\end{array}$} & \multicolumn{2}{|c|}{$\begin{array}{c}\mathrm{C} \\
\text { Batterie }\end{array}$} & \multicolumn{2}{|c|}{$\begin{array}{c}\text { D } \\
\text { Vaisselle }\end{array}$} & \multicolumn{2}{|c|}{$\begin{array}{c}\mathrm{E} \\
\text { Autres } \\
\text { contenants }\end{array}$} & \multicolumn{2}{|c|}{$\begin{array}{c}\mathrm{F} \\
\text { Entretien } \\
\text { ménager }\end{array}$} \\
\hline & \# & $\%$ & $\#$ & $\%$ & \# & $\%$ & \# & $\%$ & \# & $\%$ & \# & $\%$ & \# & $\%$ \\
\hline Québec & 212 & 33,5 & 5 &, 8 & 65 & 10,2 & 34 & 5,4 & 100 & 15,8 & 6 & ,9 & 2 & ,3 \\
\hline Montréal & 252 & 27,1 & 6 &, 6 & 94 & 10,1 & 42 & 4,5 & 99 & 10,7 & 8 & ,9 & 3 & ,3 \\
\hline
\end{tabular}


vérifiés ne justifient pas un tel décalage et la vérification des prix d'une quinzaine d'objets de consommation courante permet d'expliquer au plus $25 \%$ des 294 livres qui séparent les deux villes ${ }^{53}$. Il faut donc chercher ailleurs réponse plus satisfaisante, soit dans une inflation démesurée à Montréal, soit dans des coûts de transport exorbitants que nous n'avons pu vérifier, soit encore dans l'intérêt marqué des Montréalais pour les investissements dans les biens mobiliers. Seule une analyse détaillée de la quantité et de la qualité de chaque catégorie de biens pourrait répondre à cette dernière possibilité. Avant de procéder à cette analyse, voyons l'ensemble des catégories de biens meubles qui, comparées entre elles, suggèrent déjà des attitudes différentes (tableau 3 ). La première de ces attitudes est l'intérêt accordé par les Montréalais aux moyens de transport, aux animaux et aux denrées qui sont moins des simples lieux de consommation que des domaines d'investissement reliés aux moyens de production ou de subsistance. Les sommes investies sont trop peu considérables pour qu'on s'y arrête longuement mais elles révèlent quand même une différence entre les deux villes. Ainsi, les denrées s'y trouvent en plus grande quantité qu'à Québec et elles sont aussi plus variées. Même phénomène dans les moyens de transport, qu'il faut relier aux animaux de trait: pour un échantillon presque deux fois plus nombreux à Québec, on a sensiblement le même nombre de harnais, de colliers, de charettes, de carrioles, de tombereaux et de traînes. Mais c'est dans la catégorie des animaux que la différence est la plus marquée. A Québec, seulement 33\% des artisans se partagent quelques vaches, quelques cochons et seulement six chevaux. A Montréal, c'est $71 \%$ d'entre eux qui possèdent des animaux: les équidés ne sont guère plus nombreux, sept en tout, mais la basse-cour est plus variée. L'importance de ces catégories confirme donc le caractère plus rural de Montréa ${ }^{54}$ en même temps qu'elle indique des investissements plus prononcés dans les domaines productifs qui leur sont le plus accessibles.

La deuxième, plus évidente que la première, est l'importance accordée par les artisans québécois à l'équipement domestique par rap-

53 Pour les salaires et les prix nous avons consulté C. Nish, Les bourgeois-gentilshommes de la Nouvelle-France 1729-1748 (Montréal, Fides, 1968), 40-44; J. Mathieu, La construction navale à Québec (Québec, Société historique de Québec, 1971), 95 ss; H. A. Innis, Select Documents in Canadian Economic History, 1497-1783 (Toronto, University of Toronto Press, 1929); A. Shortt, Documents relatifs à la monnaie, aux changes et aux finances du Canada sous le Régime français (Ottawa, Imprimeur du Roi, 1925), 2 volumes. Pour ce qui est des prix, les objets suivants ont été comparés entre les deux villes: l'argenterie (48 livres le marc), les poêles en fonte, en brique et en tôle; quelques ustensiles (marmites, poêlons en cuivre, chaudrons, poêles à frire); quelques meubles (buffet en pin, armoires en pin à deux panneaux, commodes en noyer) et quelques données telles que la livre de lard salé, la livre de beurre et le minot de blé. A l'exception de l'argenterie dont le prix est fixe à 48 livres le marc durant toute la période et les poêles de fonte qui sont $25 \%$ plus chers à Montréal, les autres produits fluctuent trop, tantôt en faveur de Montréal, tantôt de Québec pour apprécier plus justement la différence de prix entre les deux villes.

54 A noter que les animaux situés en dehors de la ville, sur les terres, ne figurent pas dans ces calculs. 
port à leur avoir mobilier global. En fait, cette catégorie accapare 33,5\% des investissements mobiliers contre $27,1 \%$ à Montréal. Cette préférence pour des biens d'usage courant, des biens essentiellement de consommation est un trait distinctif des artisans de Québec qui mérite d'être explicité et le tableau 4 apporte un premier éclaircissement: peu de différence entre les deux villes dans l'éclairage, le chauffage, la batterie (toujours en proportion de l'avoir global et non en qualité) mais beaucoup dans la vaisselle, le seul secteur où Québec égale Montréal en valeur absolue et le dépasse en valeur proportionnelle $(15,8 \%$ contre $10,7 \%$ ). Toutefois, les valeurs à elles seules disent trop peu de la culture matérielle; elles ignorent tout de la quantité et de la qualité des objets, de la nature des matériaux et ne sont guère plus bavardes sur l'utilisation qu'on en faisait. Ce sont pourtant autant d'éléments qui caractérisent la culture de la vie quotidienne et qu'il nous faut considérer.

\section{A - Le feu: la cheminée et le poêle}

Dans la majorité des habitations rurales et urbaines du $17 \mathrm{e}$ siècle, la cheminée, ou le foyer comme on l'appellera plus tard, assume des fonctions multiples. D'elle dépendent en effet le chauffage, la cuisine, l'éclairage et même le repos puisque c'est tout près d'elle qu'on se rassemble la journée terminée. Et au 18e siècle, elle continue de rendre les mêmes services bien qu'elle soit de plus en plus secondée par le poêle. La quantité d'ustensiles de cheminée tels que chenets, crémaillères, trépieds, pinces et pelles à feu atteste en effet de sa présence dans presque toutes les habitations des artisans, ce qui va de soi dans un pays comme le Canada, mais elle révèle aussi qu'on l'utilise plus dans la capitale qu'à Montréal, sans doute parce qu'on y construit davantage en pierre.

Le poêle qu'on retrouve dès le $17 \mathrm{e}$ siècle dans les habitations des plus aisés, se répand au $18 \mathrm{e}$ siècle dans tous les milieux, devançant ainsi de quelques décennies la coutume française ${ }^{55}$. Étant généralement placé dans une autre pièce que le foyer ${ }^{56}$, le poêle accroît l'aire d'habitation où l'on peut vaquer à l'aise à ses occupations quotidiennes tout en décongestionnant le cercle immédiat du foyer ouvert. En outre, il rayonne mieux et permet une économie substantielle de combustible. Toutefois, à cause de son prix il n'entre pas pareillement dans tous les foyers et d'aucuns sont contraints de le louer pour la saison hivernale. A Québec, où on utilise davantage la cheminée, on compte plus de $20 \%$ des familles qui ne possèdent pas de poêle alors qu'à Montréal, c'est moins de $10 \%$. Ce sont évidemment les plus démunis des deux villes qui sont contraints de se satisfaire du foyer, beaucoup moins efficace pour le chauffage que pour la cuisine.

55 F. Braudel, Civilisation matérielle, économie et capitalisme, XVe-XVIIIe siècle, I: 262 et D. Roche, Le peuple de Paris, 142.

${ }_{56}$ L. Vermette, «Les feux domestiques...», 36. 
L'aisance matérielle n'influe pas seulement sur le nombre mais aussi sur la qualité du poêle: on y trouve le poêle en fer (ou en fonte) dont le prix varie entre 70 et 130 livres; le poêle en tôle, qui se vend en moyenne 40 livres et enfin, le moins apprécié des artisans et aussi le moins cher, le poêle de brique - simple boîte rectangulaire surmontée d'une plaque de métal - évalué à quelque 20 livres dont la moitié pour la seule plaque. Les deux derniers modèles se retrouvent tantôt chez les plus pauvres des artisans comme poêle unique, tantôt chez les plus aisés comme deuxième et même troisième poêle. Tel est le cas, par exemple des tonneliers Filion, Paquet et Normandeau de Québec ou encore des menuisiers Renaud et Jourdain-Labrosse de Montréal qui possèdent, en plus de leur poêle de fer «à la samaritaine» ou «à la palme», un poêle de brique ${ }^{57}$.

Dans les deux villes, la proportion de la valeur des biens mobiliers consacrée au chauffage est sensiblement la même, $10,1 \%$ et $10,2 \%$, mais les sommes varient: 94 livres à Montréal contre 65 livres à Québec. Certes, les inventaires nous parlent d'un plus grand nombre de maisons sans poêle à Québec en même temps que d'une utilisation plus grande des cheminées mais ils ne disent rien des familles qui se satisfont des locations saisonnières. Il ne faut donc pas conclure trop tôt à un confort plus marqué de la maison montréalaise même si des sommes plus considérables y sont consacrées au chauffage. Celles-ci s'expliquent en majeure partie par le prix du métal - fer et tôle - de quelque $25 \%$ plus élevé à Montréal.

\section{B - La batterie de cuisine et la vaisselle}

La batterie de cuisine, les ustensiles et la vaisselle de table diffèrent peu d'une ville à l'autre quant au nombre et à la variété des pièces qui composent ces catégories. La différence la plus notable vient des sommes investies de part et d'autre: dans la batterie de cuisine, la variation est peu significative, 42 livres à Montréal contre 34 livres à Québec, et nous l'avons dit, le prix plus élevé du métal à Montréal la justifie aisément; dans le cas de la vaisselle, c'est l'inverse qui se produit et on verra plus loin que la qualité des objets joue davantage que la quantité. Il y a bien quelques autres différences mineures qui ont trait à la fréquence des objets, à leur absence même, tel le fer à anguille propre à la région québécoise mais dans l'ensemble, les similitudes l'emportent sur les différences. Retenons parmi les similitudes celles qui parlent des matériaux, de la technologie des cuisines, de l'art culinaire et même des coutumes de table.

Le cuivre, le fer et la tôle sont, par ordre d'importance, les matériaux les plus usuels pour la batterie de cuisine et ce sont les mêmes

57 ANQ, Gr. J.-C. Panet, 21 juin 1752 et 29 juillet 1750, G. Boucaut de Godefus, 23 mars 1744; ANQ-M, Gr. Danré de Blanzy, 24 mai 1746 et Gr. Dufresne, 2 décembre 1744. 
TABLEAU 5

Vaisselle: comparaison entre les deux villes et

entre fortunés et moins fortunés, 1740-1755

\begin{tabular}{|l|l|c|c|c|c|c|c|c|c|c|c|c|c|}
\hline & Plats & $\begin{array}{c}\text { Assiet- } \\
\text { tes }\end{array}$ & $\begin{array}{c}\text { Écuel- } \\
\text { les }\end{array}$ & $\begin{array}{c}\text { Gobe- } \\
\text { lets }\end{array}$ & $\begin{array}{c}\text { Tas- } \\
\text { ses }\end{array}$ & $\begin{array}{c}\text { Cou- } \\
\text { teaux }\end{array}$ & $\begin{array}{c}\text { Four- } \\
\text { chettes }\end{array}$ & $\begin{array}{c}\text { Cuil- } \\
\text { lers }\end{array}$ & Pots & $\begin{array}{c}\text { Poi- } \\
\text { vrières }\end{array}$ & $\begin{array}{c}\text { Saliè- } \\
\text { res }\end{array}$ & $\begin{array}{c}\text { Ca- } \\
\text { ves }\end{array}$ & $\begin{array}{c}\text { Bou- } \\
\text { teilles }\end{array}$ \\
\hline $\begin{array}{l}\text { Québec } \\
\text { Montréal }\end{array}$ & 5 & 18,7 &, 7 & 2,4 & 1,3 & 1,2 & 6,1 & 6,3 & 2,1 &, 2 & 1, &, 5 & 4,7 \\
\hline Québec & & &, 6 & 4 & 1,1 &, 1 & 6,3 & & 1,4 &, 2 &, 4 &, 3 & 2,7 \\
Riches* & 7,8 & 28,5 &, 9 & 3,9 & 2,6 & 2,9 & 8,2 & 7,2 & 3,7 &, 4 & 1,9 & 1, & 9,5 \\
Pauvres & 2,8 & 10 &, 5 & 1,1 &, 3 &, 5 & 4,5 & 5, &, 6 & 0 &, 3 & 0 & 1,4 \\
\hline $\begin{array}{l}\text { Montréal } \\
\text { Riches* }\end{array}$ & - & - &, 8 & 4,3 & 2,7 & 0 & 7,2 & - & 3,2 & 0 &, 3 &, 5 & 2,8 \\
Pauvres & - & - &, 5 & 2,4 &, 1 &, 1 & 5,8 & - & 1, &, 1 &, 2 &, 1 & 1,3 \\
\hline
\end{tabular}

* La distinction entre les deux groupes s'est faite sur la base de la moyenne générale de la valeur des biens mobiliers. 
qu'on retrouve en France au milieu du siècle ${ }^{58}$. Le cuivre apparaît plus noble que le fer mais les plus riches se distinguent davantage par l'accumulation d'objets que par la préférence de l'un ou l'autre de ces métaux.

L'Europe du $18 \mathrm{e}$ siècle connaît surtout trois modes de cuisson: la flamme directe, la flamme indirecte et l'immersion. Ces trois procédés sont ici utilisés dans tous les foyers quoique de façon inégale. En France la cuisine à l'étouffée, à l'aide de tourtières et de braisières, est l'apanage des élites de sorte que peu d'artisans y ont recours. Ici, les braisières sont rares mais les tourtières fréquentes: chez les plus aisés, on en compte souvent plus d'une.

Le regroupement des différentes pièces de la batterie de cuisine en fonction de ces procédés aurait pu nous indiquer les préférences des artisans n'eût été de la présence de quelques ustensiles à fonctions multiples. Par exemple, le gril sert généralement à faire rôtir les viandes directement sur le feu mais il peut également servir de support à la poêle à frire; de même la chaudière en cuivre peut à l'occasion remplacer la bouilloire et le coquemar, deux ustensiles rares en cette première moitié du siècle, du moins chez les artisans. Malgré tout, il apparaît clairement que l'immersion et, à un moindre degré, la flamme indirecte sont les procédés les plus répandus et que l'utilisation de la broche à rôtir est au contraire réservée aux mieux nantis. A Québec, ce sont chez les maçons et les tonneliers et à Montréal, chez les cordonniers et les forgerons qu'on en retrouve le plus. Quelques études faites en France confirment qu'effectivement le tourne-broche est réservé aux plus aisés ${ }^{59}$.

En ce qui a trait à la vaisselle de table, la situation est fort différente: la quantité autant que la qualité des objets reflètent clairement la hiérarchie des fortunes. La comparaison entre les deux villes (tableau 5) suggère que dans l'ensemble la capitale est légèrement mieux équipée, mais cette comparaison n'est pas tout à fait concluante car la vaisselle d'étain, si populaire encore et peu chère, n'a pas reçu la même attention de la part des notaires. A Montréal, la quantité de pièces d'étain n'est précisée qu'à l'occasion alors que l'équivalent en poids l'est presque toujours ${ }^{60}$. A Québec, c'est l'inverse: le nombre de pièces est men-

\footnotetext{
$58 \quad$ Le cuivre domine dans les poêlons, les casseroles, les chaudrons, les chaudières, les tourtières, les réchauds, les écumoires et les passoires; le fer dans les grils, les broches à rôtir, les poêles à frire, les marmites et les cuillers à pot; la tôle sert surtout aux lèchefrites et aux entonnoirs mais cafetières et seaux sont parfois fabriqués avec ce matériau qu'on confond de temps à autres avec le fer-blanc.

59 Voir ces études citées dans G. Proulx, Aubergistes et cabaretiers de Louisbourg 17131758 (Parcs Canada, travail inédit no 136, 1972), 50. L'auteur avance en outre que les aubergistes et les cabaretiers de Louisbourg font un usage fréquent du tourne-broche.

60 On dira par exemple que François Gacien possède «vingt neuf livres et demie de vaisselle d'étain estimée à vingt sols la livre consistant en assiettes, plats et cuillers et le tout ensemble estimé vingt neuf livres dix sols». ANQ-M, Gr. F. Simonet, 16 mars 1745.
} 
tionné mais pas toujours l'équivalent en poids. Toute comptabilité rigoureuse des pièces d'étain est donc interdite même si les quelques données disponibles pour Montréal parlent plus de similitude que de différence. Ailleurs par contre, dans le cas des couteaux de table, des pots, des salières, et des bouteilles par exemple, l'accumulation semble légèrement plus marquée à Québec.

C'est plutôt entre riches et moins riches que les écarts sont considérables et ce dans les deux villes. Pour les objets essentiels, les quantités vont du simple au double; pour les autres, elles vont du simple au triple. La simplicité du couvert des plus démunis - une assiette, une fourchette, une cuiller en moyenne par personne ${ }^{61}$ - étonne d'autant plus qu'à l'accumulation des plus aisés, limitée il est vrai, s'ajoutent une plus grande variété et des articles de meilleure qualité.

La variété, que nos tableaux n'illustrent pas, c'est le saladier, la soupière, le sucrier, le moutardier, le huilier et même les verres qui ne font sans doute pas partie du couvert de tous les jours. Cette variété, encore modeste, ainsi que la rareté des couteaux de table, particulièrement à Montréal, ne se comprennent que par le contexte européen de l'évolution des coutumes de table. Celles-ci connaissent en effet des modifications importantes qui prennent racine chez les riches puis se propagent, parfois avec lenteur, dans la masse entre le $16 \mathrm{e}$ et le $18 \mathrm{e}$ siècles. Ainsi en est-il de la vaisselle qui connaît des usages de plus en plus spécifiques au 18e siècle; de la fourchette, un luxe encore au $17 \mathrm{e}$ siècle, qui ne se répand qu'au 18e (l'usage en devient général que vers 1750 en Angleterre); de la cuiller et du couteau individuel, dont l'habitude de les fournir se généralise dès le 16e siècle chez les riches mais dans les classes populaires, nombreux sont ceux qui, encore au $18 \mathrm{e}$ siècle, utilisent leur couteau de poche ${ }^{62}$.

Dans la Nouve:lle-France du 18e siècle, peu de différences notables avec la mère patrie: le couteau fait partie du couvert des plus riches des artisans seulement et, disons-le, ce n'est pas la règle. $\mathrm{P}$. Kalm qui fréquente surtout «les gens de qualité», dira que «le couvert de chaque personne se compose d'une serviette, d'une cuillère et d'une fourchette. On donne des couteaux quelquefois, mais en général on les omet, chaque dame et monsieur ayant soin d'apporter son propre couteau $"{ }^{63}$. Les inventaires des artisans confirment que cette coutume est généralisée; on y dénombre en moyenne pas moins de six cuillers et fourchettes par famille mais le plus souvent un seul couteau de table. En fait, quelques familles seulement en possèdent et ce sont les plus riches. Dans un pays

61 Le nombre moyen d'assiettes qui figure au tableau 5 est supérieur à un, mais presque partout, une bonne moitié des assiettes sont d' «étain vieux à refondre», donc inutilisables.

62 Sur les coutumes de table en Europe et en France, voir F. Braudel, op. cit., 174-176; N. Elias, La civilisation des moeurs (Paris, 1973) et D. Roche, op . cit., 144-148.

63 P. Kalm, Voyages..., 138. 
où les viandes fraîches ne sont pas rares, rôties ou bouillies, on comprend mieux que fourchettes et cuillers soient des ustensiles communs, même si la présence de quelques bassins et de nombreuses serviettes suppose qu'on mange encore avec ses mains.

L'autre élément de différenciation entre fortunés et moins fortunés, c'est la qualité des articles de table. L'étain et les céramiques telles que les poteries de grès ou de terre, vernissées, faïencées ou crues sont des matériaux communs qu'on retrouve partout. Impossible de dire si l'étain devance les céramiques, l'imprécision de certains inventaires dicte la prudence. Un changement se dessine toutefois, qui mérite d'être vérifié: on voit les céramiques de toutes sortes, avec une prédominance de faïence chez les plus aisés, se substituer graduellement à l'étain, suivant en cela le courant français. Plus on approche le milieu du siècle, plus nombreuses se font les mentions «d'étain vieux et usé» et «d'étain à refondre».

Le verre et le cristal, sous forme de verre de table, de gobelet, de salière ou de cave, sont des matériaux qui ornent les tables des plus aisés, sans distinction apparente entre les deux villes. L'argent, matériau encore plus précieux, entre dans cette catégorie et c'est à Québec, là où l'avoir mobilier global est moindre, qu'on le retrouve pourtant en plus grande quantité. Est-ce le signe d'une plus grande aisance camouflée par l'inégalité des prix entre les deux villes ou simplement le reflet d'un intérêt plus poussé pour la table, les réceptions ou encore le luxe et l'ostentation? Il faut jauger ces hypothèses en chiffrant l'argenterie de table dont le coût demeure stable durant cette période (48 livres le marc).

A Québec comme à Montréal, un peu plus de la moitié des artisans (respectivement $54 \%$ et $52 \%$ ) possèdent des pièces d'argenterie pour une valeur moyenne de 125 livres. L'essentiel de cette argenterie se compose de gobelets, d'assiettes, de tasses, de fourchettes et de cuillers; bijoux, montres et tabatières sont rares. Le gobelet, poinçon de Québec, sans doute témoin d'une aspiration plus que d'un signe d'aisance, est l'objet le plus commun et souvent unique chez les plus pauvres des possédants. Chez les plus aisés, l'argenterie proviendra de préférence de la métropole et le couvert (comprenant de 8 à 10 cuillers et fourchettes) semble le plus recherché avec la cuiller à potage dont le poids n'est jamais inférieur à un marc. Riches et moins riches étalent donc les mêmes symboles de richesse dans les deux villes mais les similitudes s'estompent dès qu'on examine d'un peu plus près la distribution de cet avoir. A Montréal, 77\% de la valeur globale de l'argenterie (1 055 livres sur 1375 livres) se retrouve dans les mains de quatre individus seulement dont les avoirs varient entre 100 et 350 livres. Les autres en possèdent tous pour moins de 100 livres, le plus souvent sous forme de quelques gobelets. A l'exception du cordonnier Pierre Bros- 
sard dont les 348 livres d'argenterie représentent $36 \%$ de son avoir mobilier $^{64}$, la part des autres se situe aux alentours de 15 à $20 \%$.

Québec connaît une concentration semblable où cinq individus se partagent $80 \%$ de la valeur globale de l'argenterie (2 076 sur 2590 livres). La valeur de chacun est beaucoup plus élevée qu'à Montréal, entre 310 livres et 576 livres, et prend conséquemment une plus large part de l'avoir mobilier global. La vaisselle et les ustensiles du maçon Michel Jourdain, évalués à 488 livres, représentent $38 \%$ de ses biens meubles et les 576 livres en couverts et gobelets du tonnelier Louis Paquet comptent pour $45 \%$ de ses biens mobiliers ${ }^{65}$. Pour les autres, l'argenterie ne vaut pas moins de $25 \%$ des biens meubles. La concentration à Québec est d'autant plus forte que tous les autres possédants en ont pour des sommes inférieures à ceux de Montréal, soit entre 10 et 50 livres.

A quoi donc attribuer cet intérêt des plus riches artisans québécois pour l'argenterie de table? Il serait évidemment facile d'imputer cette tendance au goût marqué des Québécois pour le luxe, l'ostentation, bref, le paraître. Les témoignages des contemporains abondent en ce sens et la littérature historique y a trouvé son compte, en s'adressant toutefois à tous les habitants sans distinction de lieu. Les intendants se réfèrent souvent à la vanité des Canadiens et plus particulièrement à leur penchant pour les belles tenues vestimentaires. Le père Charlevoix souligne le même trait en disant «qu'on y aime à se faire honneur de son bien... et qu'on fait bonne chère, si avec cela on peut avoir de quoi se bien mettre... ${ }^{66}$. P. Kalm est encore plus explicite lorsqu'il compare Montréal à Québec. A la modestie et à la simplicité des Montréalaises, il opposera l'oisiveté, la frivolité mais aussi les bonnes manières des Québécoises, influencées en cela par «des personnes appartenant à la noblesse, qui viennent chaque année de France, à bord des vaisseaux du roi, passer plusieurs semaines» alors qu'à «Montréal, on ne reçoit que rarement la visite d'hôtes aussi distingués». Des marchands, il remarque lui aussi qu'ils «s'habillent fort élégamment et poussent la somptuosité dans les repas jusqu'à la folie» ${ }^{67}$.

Il est possible que ces jugements contiennent une part de vérité et qu'ils puissent s'adresser aux plus aisés des couches populaires, les artisans entre autres, souvent enclins à imiter les modes métropolitaines et à adopter certains comportements des bourgeois et des seigneurs. Bien dresser une table avec une dizaine de «couverts» en argent, des gobelets ou des verres et à l'occasion un chandelier au centre peut sûre-

64 ANQ-M, Gr. L.CC. Danré de Blanzy, 26 septembre 1743.

65 ANQ, Gr. C. Barolet, 10 novembre 1752 et Gr. J.-C. Panet, 29 juillet 1750.

66 Père F.-X. Charlevoix, Histoire et description générale de la Nouvelle-France (Paris, 1744), V: 117

67 P. Kalm, Voyages..., 43, 81, 103, 214-215. 
ment impressionner les convives tout en faisant l'orgueil du propriétaire. D'ailleurs, la quantité impressionnante de nappes et de serviettes inventoriées dans les foyers des plus aisés tend à confirmer l'intérêt des Québécois pour les plaisirs de la table sinon leur goût pour l'apparat ${ }^{68}$. Par ailleurs, il apparaît bien plus probable que dans une période d'insécurité et de rareté de monnaie métallique, l'argenterie soit considérée d'abord et avant tout comme un gage de sécurité, un objet de thésaurisation ou un simple moyen d'accroître son patrimoine au même titre que le numéraire. Les quelques legs aux héritiers tantôt d'une cuiller tantôt d'une assiette et la fréquente mention d'une seule pièce d'argenterie, le gobelet, dans les foyers les plus modestes tendent à confirmer notre hypothèse. Et précisons-le, les artisans québécois affichent en ce domaine une tendance plus marquée, tendance qui, nous l'avons vu, se manifeste également dans la large part que prend le numéraire dans leur fortune globale.

\section{C - Le mobilier}

Pris dans son acception actuelle, le mobilier est sans doute le domaine qui donne l'accès le plus direct au cadre de vie quotidien des artisans. C'est aussi un domaine qui reflète bien les manières de consommer: le nécessaire étant assuré, entendons surtout les meubles essentiellement utilitaires, les plus fortunés peuvent se permettre de multiplier les meubles de rangement, les chaises, les tables ou d'ajouter quelques objets de décor. En divisant le mobilier en trois catégories, les meubles meublant, les objets de décor et le lit et la literie, nous serons donc en mesure de distinguer le nécessaire du superflu et de compléter ainsi le portrait de l'univers quotidien des familles d'artisan.

Le mobilier accapare dans les deux villes $25 \%$ de la valeur totale des biens meubles ${ }^{69}$. Encore une fois, la similitude entre les deux villes est patente: les montants investis ne sont pas les mêmes mais la part de la fortune mobilière consacrée aux meubles est comparable. De même, fortunés et moins fortunés connaissent des écarts considérables, et ceuxci sont plus prononcés à Québec, particulièrement dans les investissements reliés aux objets de décor et à la chambre à coucher ${ }^{70}$. Mais n'insistons pas outre mesure sur ces écarts révélés par les seules valeurs;

68 On dénombre effectivement une moyenne de 13,5 nappes et 34,8 serviettes par foyer à Québec, alors qu'à Montréal, ces moyennes tombent respectivement à 11,4 et 17,7 par foyer.

69 Cette proportion s'apparente à celles de la France rurale et urbaine du $18 \mathrm{e}$ siècle. Pour la ville, voir D. Roche, op . cit.; pour le monde rural, voir J. Peret, «Les meubles ruraux en HautPoitou au XVIIIe siècle d'après les inventaires après décès», Évolution et éclatement du monde rural, France Québec XVIIe-XXe siècles (Montréal et Paris, Presses de l'Université de Montréal et École des hautes études en sciences sociales, 1986), 487-498; et M. Baulant, "Niveaux de vie paysans autour de Meaux en 1700 et 1750», Annales ESC, 2-3 (1975): 505-518.

${ }_{70}$ En fait, les sommes consenties par les plus fortunés de la capitale à ces deux domaines sont respectivement quinze et cinq fois plus élevées que celles qu'y consacrent les moins fortunés. A Montréal, ces proportions chutent respectivement à cinq et trois et confirment la meilleure distribution des fortunes annoncée dès le début de cette analyse. 
TABLEAU 6

Mobilier: comparaison entre les deux villes et entre fortunés et moins fortunés, $\mathbf{1 7 4 0 - 1 7 5 5}$

\begin{tabular}{|c|c|c|c|c|c|c|c|c|c|c|c|c|c|c|c|c|}
\hline & \multicolumn{10}{|c|}{ Meubles meublants } & \multicolumn{6}{|c|}{ Objets de décor } \\
\hline & $\begin{array}{l}\text { ta- } \\
\text { bles }\end{array}$ & $\begin{array}{c}\text { chai- } \\
\text { ses }\end{array}$ & $\begin{array}{l}\text { fau- } \\
\text { teuils }\end{array}$ & $\begin{array}{l}\text { ber- } \\
\text { gères }\end{array}$ & $\begin{array}{l}\text { tabou- } \\
\text { rets }\end{array}$ & $\begin{array}{l}\text { armoi- } \\
\text { res }\end{array}$ & $\begin{array}{l}\text { buf- } \\
\text { fets }\end{array}$ & $\begin{array}{l}\text { com- } \\
\text { modes }\end{array}$ & $\begin{array}{l}\text { cof- } \\
\text { fres }\end{array}$ & $\begin{array}{l}\text { hu- } \\
\text { ches }\end{array}$ & $\begin{array}{l}\text { mi- } \\
\text { roirs }\end{array}$ & $\begin{array}{l}\text { ca- } \\
\text { dres }\end{array}$ & $\begin{array}{c}\text { ima- } \\
\text { ges }\end{array}$ & $\begin{array}{l}\text { tapis- } \\
\text { series }\end{array}$ & $\begin{array}{l}\text { ta- } \\
\text { pis }\end{array}$ & $\begin{array}{c}\text { ri- } \\
\text { deaux }\end{array}$ \\
\hline Québec & 1,9 & 11,8 &, 5 &, 1 &, 7 &, 5 &, 7 &, 2 & 1,1 &, 7 & 1,0 & 1,3 & 1,2 & ,2 & ,3 & 1,0 \\
\hline $\begin{array}{l}\text { Montréal } \\
\text { Québec }\end{array}$ & 2,0 & 9,4 &, 8 &, 2 &, 4 &, 7 &,$\overline{8}$ &, 1 &, 5 &, 7 & ,9 &, 6 &, 1 &, 3 & 0 &, 5 \\
\hline Riches* & 2,3 & 15,7 &, 7 &, 1 & 1,0 &, 7 & ,8 &, 4 & 1,5 & ,8 & 1,8 & 1,6 & 2,7 & 6 & ,6 & 1,9 \\
\hline $\begin{array}{l}\text { Pauvres } \\
\text { Montréal }\end{array}$ & 1,6 & 9,1 &, 4 & 0 &, 5 &, 3 & 6 &, 1 &, 8 &, 7 &, 5 & 1,0 & 0 & 0 & 0 &, 4 \\
\hline Riches* & 2,2 & 10,7 & 1,2 &, 1 & ,4 & ,8 & 6 & ,3 &, 4 &, 7 & 1,3 & 1,3 &, 1 &, 7 & 0 & ,9 \\
\hline Pauvres & 1,8 & 8,2 &, 4 &, 3 &, 4 &, 5 & ,9 & 0 & ,6 & ,9 &, 6 & 0 & 0 & 0 & 0 & ,2 \\
\hline
\end{tabular}

\section{Lit et literie}

\begin{tabular}{|c|c|c|c|c|c|c|c|c|c|c|c|}
\hline & $\begin{array}{l}\text { cou- } \\
\text { chettes }\end{array}$ & $\begin{array}{l}\text { lits de } \\
\text { plumes }\end{array}$ & $\begin{array}{l}\text { pail- } \\
\text { lasses }\end{array}$ & $\begin{array}{l}\text { mate- } \\
\text { las }\end{array}$ & $\begin{array}{l}\text { tours } \\
\text { de lit }\end{array}$ & $\begin{array}{c}\text { cou- } \\
\text { vertes }\end{array}$ & $\begin{array}{l}\text { courte- } \\
\text { pointes }\end{array}$ & draps & $\begin{array}{c}\text { traver- } \\
\text { sins }\end{array}$ & $\begin{array}{c}\text { oreil- } \\
\text { lers }\end{array}$ & $\begin{array}{c}\text { taies } \\
\text { d'oreillers }\end{array}$ \\
\hline Québec & 1.6 & 2.5 & 2.0 & .5 & .6 & 3.1 & 1.1 & 7.7 & 1.4 & 1.7 & .8 \\
\hline Montréal & 1.2 & 2.0 & 1.6 & .2 & .4 & 2.6 & .8 & 7.7 & .7 & 1.4 & 1.1 \\
\hline \multicolumn{12}{|l|}{ Québec } \\
\hline Riches* & 2.4 & 3.8 & 2.8 & .8 & .9 & 4.4 & 1.7 & 14.7 & 1.9 & 2.6 & 1.8 \\
\hline Pauvres & 1.2 & 1.6 & 1.4 & .3 & .3 & 2.1 & .6 & 2.8 & 1.1 & 1.1 & .1 \\
\hline \multicolumn{12}{|l|}{ Montréal } \\
\hline Riches* & 1.6 & 2.2 & 1.9 & .4 & .6 & 3.2 & 1.0 & 12.2 & .6 & 2.1 & 2.2 \\
\hline Pauvres & 1.0 & 1.8 & 1.4 & .0 & .2 & 2.2 & .6 & 3.6 & .8 & .8 & .2 \\
\hline
\end{tabular}

* La distinction entre les deux groupes s'est faite sur la base de la moyenne générale de la valeur des biens mobiliers. 
retenons plutôt le nombre, la fonction, la variété et l'utilisation de ces biens mobiliers et voyons en quoi se distinguent fortunés et moins fortunés.

Tous sont bien pourvus en meubles de groupe, nécessaires à la sociabilité: deux tables en moyenne par famille, une grande de facture simple pour les repas et une autre petite, parfois rangée dans un coin, et entre 8 et 12 chaises, quelques-unes de plus chez les plus aisés (tableau 6). Aucune mention de banc chez ces citadins, une absence qui témoigne de l'individualisation du mobilier tout comme en France à la même époque. Les fauteuils, sièges généralement de luxe qui se multiplient dans la France urbaine du 18e siècle, ne sont ici que de pâles imitations de ce que l'on rencontre dans les classes supérieures de la société. Point de fauteuil rembourré et garni de tissu mais de simples fauteuils «foncés» de bois de pin, de merisier ou à fond tressé. Simples mais plus confortables que les traditionnelles chaises droites, ces sièges sont deux fois plus nombreux chez les plus aisés, signe que le confort, même le plus modeste, n'entre pas pareillement partout. Et c'est à Montréal qu'on les retrouve en plus grand nombre, avec quelques bergères, fauteuils plus larges et plus profonds.

Aucune succession n'est privée du mobilier nécessaire à l'ordonnance des choses mais les manières de ranger peuvent différer. L'armoire, le buffet et le coffre sont les meubles de rangement les plus communs. Contrairement à ce que l'on pourrait croire, l'armoire n'est pas le meuble qui fait partie de toutes les maisons urbaines. A Québec, une seule famille sur deux en possède alors qu'à Montréal c'est deux sur trois. A cette armoire mobile il faut toutefois ajouter celle encastrée dans le mur, fabriquée à même la structure de la maison, plus fréquente à Québec, là où les structures de maçonnerie dominent. Le buffet, un meuble qui s'apparente à l'armoire mais en plus bas, est presque aussi populaire dans les deux villes et ce sont les moins fortunés de Montréal qui en possèdent davantage. Il faut dire aussi qu'il est presque toujours deux fois moins cher que l'armoire ${ }^{71}$. Ces deux meubles, répondant à une même forme de rangement ancien qu'est l'empilement sur des étagères, se retrouvent dans une majorité de foyers.

Le coffre, rustique et de fabrication facile, est le meuble des errants, des mobiles, celui qu'on amène aisément avec soi dans les déménagements. Dans la France rurale, on en compte en moyenne trois par foyer mais en milieu urbain, du moins à Paris, il est graduellement remplacé par le buffet ${ }^{72}$. Une semblable tendance semble se dessiner ici: les buffets sont nombreux, plus nombreux même que l'armoire, alors que le

71 La popularité du buffet au 18e siècle est confirmée dans l'enquête de Jocelyne Mathieu, «Le mobilier contenant», BHCM, 20 (automne 1984): 1-18.

72 D. Roche, op. cit., J. Peret, op. cit., et S. Tardieu, Le mobilier rural traditionnel français (Paris, Aubier-Flammarion, 1976). 
coffre ne se retrouve même pas dans tous les foyers. A Montréal, il est présent dans un foyer sur deux; à Québec, dans un sur trois seulement chez les plus pauvres mais partout chez les plus aisés. Sa fréquence chez ces derniers s'explique sans doute par un plus grand besoin de meubles de rangement qu'exige l'accumulation de biens de toutes sortes qu'on ne veut pas nécessairement exhiber. Le coffre n'est-il pas le plus vieux meuble de rangement que l'on connaisse et dans lequel on entasse linge de maison, vêtements et tissus qu'on sortira au besoin?

Fonctionnels, essentiellement utilitaires, sobres, telles sont les caractéristiques dominantes de ces trois meubles qui réunissent pour un temps fortunés et moins fortunés. Quant à la commode, meuble essentiellement urbain, qui allie le fonctionnel à l'apparat, elle demeure l'apanage de quelques artisans fortunés seulement qui aspirent aux goûts et aux modes des couches supérieures de la société. Il faudra attendre le $19 \mathrm{e}$ siècle avant qu'elle ne concurrence sérieusement l'armoire.

Plus que le meuble fonctionnel, même celui bien fait, bien stylisé, ce sont les objets de décor qui peuvent le mieux définir l'ambiance de ces foyers. Dans la maison des plus humbles, le décor se résume à quelques rideaux de toile habillant fenêtres et portes, à quelques cadres et à quelques miroirs dans moins de la moitié des foyers; dans l'ensemble, c'est une nudité surprenante qui caractérise l'atmosphère de près des deux tiers de ces maisons. Ajoutons si l'on veut quelques crucifix que les estimateurs auraient volontairement oubliés, quelques bibelots placés ici et là sur les meubles et les tablettes, le contraste avec le décor des plus fortunés n'en demeure pas moins frappant et, une fois de plus, davantage à Québec qu'à Montréal.

A l'exception des tapisseries que l'on retrouve en quantité à peu près égale dans les deux villes, tous les autres objets de décor sont plus nombreux à Québec. Les cadres, les images, souvent estimées à quelques deniers seulement, les tapis et même les figurines en plâtre, les vases et les lustres de cheminés que nos tableaux ignorent, sont l'exclusivité des plus fortunés de Québec. Les inventaires sont malheureusement trop souvent avares de description mais on sait que les cadres des plus riches sont de bois doré ou "peinturés», que quelques miroirs sont encadrés, qu'il y a quelques belles tapisseries «de Bergame» et quelques vases de cheminée en faïence. C'est bien peu mais suffisant pour pouvoir imaginer le souci et surtout la possibilité qu'ont les plus fortunés seulement d'aménager de façon agréable leur espace intérieur.

La chambre à coucher est l'univers qui absorbe la plus grande part des investissements dans les biens meubles. Symbole du foyer conjugal, refuge des rapports intimes, son importance se manifeste de plusieurs façons: d'abord par son isolement des autres pièces de la maison au moyen de cloisons, ensuite par l'importante proportion de l'avoir 
mobilier que riches et moins riches des deux villes y consacrent, soit environ $16 \%$.

La comparaison entre les deux villes et entre riches et pauvres présente les mêmes caractéristiques que les autres secteurs du mobilier: les lits et les pièces de la literie sont plus nombreux à Québec qu'à Montréal et les écarts entre riches et pauvres y sont plus accentués. En fait, les plus fortunés de la capitale possèdent entre deux et trois fois plus d'objets que les moins fortunés, à commencer par les lits. A cause de l'ambiguïté terminologique de l'époque, ceux-ci sont évidemment difficiles à évaluer avec exactitude, mais en se fiant aux termes de l'inventaire ${ }^{73}$, il y aurait 1,6 couchette par foyer à Québec et 1,2 à Montréal pour une moyenne de 2,7 personnes par lit à Québec et 3,7 à Montréal $^{74}$. Dans les deux cas, la promiscuité paraît inévitable mais nous savons que celle-ci pouvait être facilement soulagée par des paillasses simplement déposées sur le plancher, coutume pratiquée dans certaines auberges $^{75}$.

Quoiqu'il en soit de ces moyennes qu'il est impossible de vérifier par d'autres sources, les écarts entre fortunés et moins fortunés sont patents. Ils affectent d'abord les rapports intimes du couple par l'exclusion des autres membres de la famille du lit conjugal: les fortunés ont deux fois plus de couchettes que les pauvres dans les deux villes et il en est de même des lits de plume, des paillasses et des matelas bien que la différence soit moins marquée à Montréal. Ils affectent ensuite le confort calorifique que le feu de l'âtre ou du poêle ne pouvait fournir en tout temps: ceux-là même qui possèdent le plus de poêles ont aussi plus de couvertes, de courtepointes et de tours de lit. Enfin, ils affectent aussi les tâches ménagères telles que le lavage et le repassage: l'accumulation de draps chez les plus fortunés le laisse aisément supposer: 14,7 contre 2,8 à Québec et 12,2 contre 3,6 à Montréal.

Au-delà de ces moyennes et de ces contrastes, il est une réalité commune à tous: chaque foyer possède au moins son lit garni. Certains paraissent sobres, tel celui du charpentier Jean Lefebvre de Québec, évalué à quelque 40-50 livres. Il comprend un «vieux châlit»en merisier, une paillasse, un lit de plume couvert de couty (coutil), un matelas de laine couvert de toile à carreaux, une paire de draps de «toile de commun», deux couvertes blanches, une bonne et une vieille, une courtepointe d'indienne et un traversin pareil au lit de plume ${ }^{76}$. Dans cette catégorie sociale, le merisier est le matériau le plus usuel pour les cou-

73 L'expression la plus commune est la couchette et le lit de plume n'est en fait qu'une sorte de matelas «que l'on étend sur toute la longueur du lit (armature) dessus la paillasse». N. Genêt et al., op. cit., 151 .

${ }_{74}$ Moyenne effectuée sur la base de 4,4 personnes par foyer à Québec et 4,5 à Montréal (moyenne tirée de nos inventaires).

75 G. Proulx, op. cit., 50ss

76 ANQ, Gr. C.-H. Dulaurent, 14 décembre 1745. 
chettes et le coton et le coutil sont les textiles les plus communs. A l'exception de la toile à carreaux qui recouvre le matelas, les tissus à motifs sont rares et le blanc ou crème domine dans les couleurs.

D'autres sont beaucoup plus complexes et reflètent bien l'aisance de certains. Celui du maçon Michel Jourdain, comprend

une couchette en merisier et sa «fonsure», une vieille paillasse de toile de «brin», un lit et un traversin de plume couvert de coutil «à grande barre», un vieux matelas de laine et crin couvert de toile à carreaux, deux draps de toile herbée, deux couvertures blanches en laine, l'une de «catalogne» l'autre de Rouen, une courtepointe en coton brodé, deux oreillers de toile peinte et un tour de lit de serge verte consistant en deux rideaux, deux grâces (bonnes-grâces) et un ciel de lit avec pentes et sous-pentes découpées en festons et ornées d'un ruban vert avec tringles et anneaux de fer. ${ }^{77}$

Un tel ensemble, qui vaut entre 100 et 150 livres, offre évidemment un contraste frappant avec celui du charpentier Lefebvre: non seulement allie-t-il confort corporel et intimité mais on y observe une plus grande variété de textiles et de couleur où le vert domine; comme dans la France populaire, on ne peut pas encore se permettre d'imiter les modes bourgeoises qui privilégient déjà les «toiles peintes à fleurs et à ramages» ${ }^{78}$. Ajoutons que les chambres à coucher des mieux nantis sont souvent munies d'un deuxième poêle en plus d'être meublées un peu à la manière des salles communes des moins fortunés, avec buffet, coffre, fauteuil, chaises et au mur, crucifix et images. Et cet arrangement n'est pas l'exclusivité de quelques nantis parmi les artisans mais est plutôt partagé par un bon tiers d'entre eux à Québec comme à Montréal.

A l'exception de quelques indigents, tous les artisans sont en général bien pourvus en meubles utilitaires. Les plus fortunés se démarquent franchement toutefois par le nombre de meubles et la qualité des matériaux dont ils sont fabriqués, par une literie plus abondante et plus variée et par un souci de décorer leur univers intérieur.

\section{CONCLUSION}

La répartition de la fortune mobilière nette des artisans des deux villes a mis en évidence une opposition bien tranchée entre petits patrimoines et successions plus importantes. D'un côté les plus démunis, environ $60 \%$ dans la capitale comme à Montréal, avec quelque 500 à 700 livres de biens mobiliers mangés pour une majorité d'entre eux par les dettes. Les patrimoines négatifs sont en effet impressionnants partout: entre 33 et $38 \%$ et c'est là un pourcentage conservateur car on sait que les inventaires n'atteignent pas toujours les plus pauvres. De l'autre

77 ANQ, Gr. C. Barolet, 10 novembre 1752.

78 D. Roche, op. cit., 136. 
les plus fortunés avec de 800 à 2000 livres de biens mobiliers, un peu plus nombreux à Montréal qu'à Québec. L'addition du numéraire, des créances, des dettes et surtout des biens fonciers et immobiliers a fait ressortir cependant que les fortunes les plus imposantes sont plus nombreuses du côté de Québec.

Il est fort possible que la source notariale avantage les aisés mais il n'en demeure pas moins qu'une importante partie de la population artisanale est propriétaire de sa maison. Beaucoup plus à Montréal qu'à Québec, notre sondage donne $90 \%$ dans la première ville contre $64 \%$ dans la deuxième. En revanche la propriété québécoise est faite de pierre, offre une surface habitable plus grande à cause de sa hauteur alors que la maison montréalaise est en bois, basse et n'a qu'un étage. La différence la plus notable réside toutefois dans le prix, de deux à trois fois plus élevé à Québec; aussi, l'avance que la seule richesse mobilière semblait conférer à Montréal disparaît au profit de Québec et ce malgré un nombre inférieur de propriétaires dans la capitale.

L'analyse sommaire d'une catégorie de biens indispensables dans tout foyer, l'équipement domestique et particulièrement la vaisselle, a révélé clairement que les artisans québécois accordent une plus grande importance que les Montréalais à l'argenterie de table. Un même pourcentage d'individus en possède dans les deux villes mais la valeur de l'argenterie concentrée dans les mains d'une minorité à Québec est plus considérable. Signe d'activités sociales plus intenses, symbole d'aisance, objet d'ostentation ou surtout de thésaurisation? La valeur de l'argenterie prend une place si grande dans l'avoir mobilier global des plus fortunés que la dernière hypothèse est la plus probable. D'ailleurs l'accumulation de numéraire chez ceux-là même qui possèdent les plus grosses valeurs en argenterie semble le confirmer.

Beaucoup plus que l'argenterie et le numéraire, les investissements fonciers et immobiliers distinguent les artisans des deux villes. L'explication réside moins dans des attitudes différentes face au capital que dans les limites qu'imposent des fortunes peu considérables et dans les possibilités qu'offre le marché local et régional. A Montréal, la terre attire plus que la maison ou l'emplacement en ville et elle apparaît de peu de valeur en comparaison des investissements immobiliers que préfèrent les Québécois. Il n'a pas été possible d'estimer à leur juste valeur les biens fonciers et immobiliers mais les évaluations que livrent les inventaires, souvent inférieures à la valeur marchande ${ }^{79}$ laissent entrevoir clairement que bon nombre d'artisans s'élèvent au-dessus d'une aisance médiocre et ce, davantage à Québec qu'à Montréal.

79 Sur le «cru» et la différence de prix entre l'estimation lors de l'inventaire et la vente des biens, voir L. Dechêne, Habitants..., 379, et le compte rendu que F. Ouellet fait du livre de L. Dechêne dans Histoire sociale/Social History, 3,16 (novembre 1975): 380-381. 
En-deçà des fortunes et des valeurs qui varient trop d'une ville à l'autre, il y a tout le domaine de la consommation, de la qualité de vie de tous les jours, bref, du confort. L'analyse quantitative et qualitative des objets nous a permis de l'explorer. Que l'on regarde le système de chauffage, la batterie de cuisine, la vaisselle ou le mobilier de la salle commune et de la chambre à coucher, l'indigence est rare et la majorité est pourvue de l'essentiel. Les écarts apparaissent toutefois partout considérables entre fortunés et moins fortunés. D'abord dans la quantité des objets et ce, particulièrement dans la chambre à coucher; ensuite dans la qualité et la variété exprimées par un plus grand choix de matériaux, de textiles et de couleurs. Enfin, de façon plus manifeste encore, dans l'atmostphère qu'on essaie d'imaginer tant bien que mal grâce aux objets de décor.

Gardons-nous cependant d'un portrait trop optimiste des artisans de Québec et de Montréal. Trop d'aspects de leur vie et de leur travail n'ont pas été abordés. La structure de leur fortune n'a été qu'effleurée, sans considérer leur revenu professionnel ni leur possibilité de travailler, de s'alimenter et de s'habiller. Riches et pauvres, il va de soi, diffèrent en ces domaines. Nous n'avons pas non plus touché à la hiérarchie des occupations qui semble jouer un rôle beaucoup plus important dans la colonie que dans la métropole à la même époque où la différence entre les fortunes «ne vient généralement pas des qualifications professionnelles» ${ }^{80}$. Sans doute, en ce domaine, le fait d'être artisan indépendant ou salarié compte pour beaucoup.

De même, la proportion impressionnante de propriétaires parmi les artisans et la qualité de leurs habitations appellent des nuances. L'inventaire après décès n'est pas la meilleure source pour évaluer la propriété. De plus, les données de Montréal excluent les faubourgs, il est vrai encore peu peuplés, et celles de Québec comptent peu d'artisans du quartier ouvrier de Saint-Roch en formation. Et que dire de l'espace habitable de ces maisons qui serait certainement corrigé à la baisse si on pouvait évaluer le taux de location, particulièrement à Québec où les propriétaires sont moins nombreux ${ }^{81}$.

Ces quelques remarques n'enlèvent rien aux traits caractéristiques des artisans des deux villes non plus qu'à la nette opposition entre pauvres et plus fortunés. L'imperfection de la source étant la même partout, elles incitent simplement à considérer chiffres et pourcentages davantage comme des ordres de grandeur que comme des données décisives et à poursuivre cette enquête sur d'autres groupes sociaux, en élargissant toutefois l'éventail des sources.

80 D. Roche, Le peuple de Paris, 88.

81 A Montréal, on sait déjà que le pourcentage de locataires est faible vers 1741: sur 457 maisons dans la ville et les faubourgs, seulement 66 logent deux familles et plus. L. Dechêne, «Croissance...», 169. 